\title{
Synchronization of inertial memristive neural networks with time-varying delays via static or dynamic event-triggered control
}

\author{
Wei Yao ${ }^{\mathrm{a}}$, Chunhua Wang ${ }^{\mathrm{a}, *}$, Yichuang Sun ${ }^{\mathrm{b}}, \mathrm{Chao}_{\mathrm{Zhou}}^{\mathrm{a}}$, Hairong Lin $^{\mathrm{a}}$ \\ ${ }^{a}$ College of Information Science and Engineering, Hunan University, Changsha 410082, \\ China \\ ${ }^{b}$ School of Engineering and Technology, University of Hertfordshire, Hatfield AL10 9AB, \\ $U . K$
}

\begin{abstract}
This paper investigates the synchronization problem of inertial memristive neural networks (IMNNs) with time-varying delays via event-triggered control (ETC) scheme and state feedback controller for the first time. First, two types of state feedback controllers are designed; the first type of controller is added to the transformational first-order system, and the second type of controller is added to the original second-order system. Next, based on each feedback controller, static event-triggered control (SETC) condition and dynamic event-triggered control (DETC) condition are presented to significantly reduce the update times of controller and decrease the computing cost. Then, some sufficient conditions are given such that synchronization of IMNNs with time-varying delays can be achieved under ETC schemes. Finally, a numerical simulation and some data analyses are given to verify the validity of the proposed results.
\end{abstract}

Keywords: Synchronization, inertial memristive neural networks, event-triggered control, state feedback controllers.

\footnotetext{
This work was supported in part by the National Natural Science Foundation of China under Grant 61971185, the Major Research Project of the National Natural Science Foundation of China under Grant 91964108 and the Open Fund Project of Key Laboratory in Hunan Universities under Grant 18K010.

* Corresponding author

Email address: wch1227164@hnu.edu.cn (Chunhua Wang)
}

Preprint submitted to Neurocomputing

April 15, 2020 


\section{Introduction}

As one of the most important dynamical characteristics of complex systems, synchronization has been widely studied [1-5]. Currently, synchronization and neural networks can be applied in many potential areas [6-10], such 5 as image encryption, biological systems and secure communication. In 2008, the memristor prototype was realized by HP lab using $\mathrm{TiO}_{2}$ [11]. Since then, memristor-based circuits and applications have been investigated extensively in the academic world [12-16]. Because of the nonvolatility of memristor, memristive neural networks (MNNs) can be built with memristor to emulate synapse. MNN has broad applications in several fields $[17,18]$, such as logical operations and image processing. Therefore, the topics of synchronization of MNNs have attracted increasing attention [19-27]. For example, Wu et al. researched a class of memristive recurrent neural networks (NNs) and achieved exponential synchronization of NNs via Lyapunov functional and differential inclusions [19].

15 In [20], global exponential synchronization of memristive recurrent NNs with time-varying delays was addressed by using Lyapunov functional method and fuzzy theory. In [21], some sufficient conditions were presented to ensure synchronization of coupled MNNs via impulsive differential inequality and extended Halanay differential inequality. Li and Cao [22] investigated lay synchronization and lag quasi-synchronization of coupled MNNs by utilizing generalized Halanay inequality and $\omega$-measure method. In [23], synchronization problem of fractional-order MNNs was addressed under some sufficient conditions.

It is worth noting that [19-27] focused primarily on the first order derivative or fractional order derivative of the state variables. Recently, the dynamical characteristic research of MNNs via introducing an inertial term has attracted great interest [28-37], because the inertial term is considered as a key tool to generate complicated chaos and bifurcation behavior. In [28], Wang et al. investigated global stabilization of inertial memristive recurrent NNs with discrete delays and two types of distributed delays. By using matrix measure method, stability and pinning synchronization of inertial MNNs (IMNNs) 
with time delays were studied in [30]. Some sufficient conditions were given in [31] such that global exponential synchronization of coupled IMNNs can be achieved via state feedback control method. In addition, by using different control methods, synchronization of IMNNs can be realized in [32-37]. For example, finite-time synchronization of IMNNs with time delay was addressed via delay-dependent control technique [32]. In [33], fixed-time synchronization and finite-time synchronization of IMNNs were achieved via state feedback control method. Similarly, fixed-time and finite-time synchronization of IMNNs were studied by fixed-time control method and state feedback control method [34], respectively. In [36], global exponential synchronization of IMNNs was investigated via nonlinear control method. Using nonlinear coupling, namely, nonlinear feedback control strategy, global exponential synchronization of multiple coupled IMNNs with time-varying delays was achieved [37]. Currently, due to some advantages including reliability and high efficiency, network control schemes such as state feedback control method and nonlinear control method, have been widely researched and applied in several areas [32-39]. However, these network control schemes used in IMNNs [32-37] are based on continuous-time feedback controllers, which means that these systems have heavy computing burden. Seriously, these continuous-time controllers may lead to congestion of communication channels.

As two sampling control schemes, time-triggered control [40, 41] and eventtriggered control (ETC) [42-50] can effectively reduce computing cost and communication resources. Nevertheless, when the consecutive sampling-data interval is infinitesimal, there exist wastefulness of computing cost and unnecessary energy consumption for time-triggered control. Fortunately, ETC can solve the problem. Unlike time-triggered control, ETC can significantly reduce update times of controller and ensure the system performance. Therefore, ETC scheme used in controlled systems has received increasing attention and some relevant topics have been studied [42-50]. In [45], Zhang et al. discussed stability 60 of MNNs with communication delays by using event-triggered sampling control method. Using event-triggered impulsive control, Zhou and Zeng realized 
quasi-synchronization of MNNs with time-varying delays [46]. In [47], an eventtriggered $H_{\infty}$ state estimation was designed and it guaranteed that delayed discrete-time stochastic MNNs were exponentially mean-square stable. Guo et al. presented two types of ETC methods for synchronization of delayed MNNs [48]. In [49], an event-triggered communication scheme is proposed to save the communication resources of nonlinear multiagent systems with unknown and nonidentical control directions. However, these ETC schemes were only considered in the first-order system [42-50], and only one type of measured error was addressed. This means the existing ETC methods cannot be directly used in the second-order system such as IMNNs which needs to consider two type$\mathrm{s}$ of measured errors. To the best of our knowledge, there is little work on synchronization of IMNNs via ETC scheme.

Inspired by the discussion above, this paper investigates the synchronization 75 of IMNNs with time-varying delays via ETC scheme for the first time. We summarize the main contributions as follows.

1) This paper designs two types of state feedback controllers. The first type of controller is added to the transformational first-order response system, and the second type of controller is added to the original second-order response system.

2) Based on each state feedback controller, two types of ETC schemes, namely, static event-triggered control (SETC) and dynamic event-triggered control (DETC) were designed.

3) Some sufficient conditions are presented to guarantee synchronization of IMNNs with time-vary delays via ETC scheme under two types of state feedback controllers.

4) Under ETC schemes, the IMNNs can effectively reduce the update times of feedback controllers and decrease computing burden. Moreover, compared with the existing ETC methods [42-50], our control schemes are more flexible.

The rest of the paper is organized as follows. In Section 2, IMNNs with timevarying delays are introduced. Two types of controllers and some ETC schemes are designed to realize synchronization of IMNNs in Section 3. Section 4 presents 
a numerical simulation and some data analyses to verify the effectiveness of the obtained results. Finally, conclusions are given in Section 5 .

\section{Preliminaries}

First, we give some notations which will be used later.

Notations: For a given vector $a=\left(a_{1}, a_{2}, \ldots, a_{l}\right)^{T},\|a\|_{1}=\sum_{m=1}^{l}\left|a_{m}\right|$. For a given matrix $x=\left[x_{m z}\right]_{l \times l},\|x\|_{1}=\max _{1 \leq z \leq l} \sum_{m=1}^{l}\left|x_{m z}\right|$, and $\lambda(x)$ represents all eigenvalues of matrix $x$.

We consider IMNNs with time-varying delays as follows.

$$
\begin{aligned}
& \frac{d^{2} p_{m}(t)}{d t^{2}}=-b_{m} \frac{d p_{m}(t)}{d t}-c_{m} p_{m}(t)+\sum_{z=1}^{l} \alpha_{m z}\left(p_{m}(t)\right) \\
& \times f_{z}\left(p_{z}(t)\right)+\sum_{z=1}^{l} \beta_{m z}\left(p_{m}(t)\right) f_{z}\left(p_{z}\left(t-\tau_{m z}(t)\right)\right) \\
& +I_{m}(t), m=1,2, \ldots, l,
\end{aligned}
$$
second order derivative of $p_{m}(t)$ is an inertial term; $\alpha_{m z}$ and $\beta_{m z}$ represent memristive connection weights; $f_{z}(\cdot)$ is the activation function. $\tau_{m z}(t)$ denotes time-varying delay and satisfies $0 \leq \tau_{m z}(t) \leq \tau$, where $\tau$ is a positive constant; $I_{m}(t)$ is external input.

We consider the initial conditions of system (1) as

$$
\left\{\begin{array}{l}
p_{m}(s)=\Upsilon_{m}(s), \\
\frac{d p_{m}(s)}{d s}=\Theta_{m}(s), \quad-\tau \leq s \leq 0 .
\end{array}\right.
$$

We set memristive connection weights as

$$
\begin{aligned}
& \alpha_{m z}\left(p_{m}(t)\right)= \begin{cases}\vec{\alpha}_{m z}, & \left|p_{m}(t)\right| \leq \chi_{m}, \\
\overleftarrow{\alpha}_{m z}, & \left|p_{m}(t)\right|>\chi_{m},\end{cases} \\
& \beta_{m z}\left(p_{m}(t)\right)= \begin{cases}\vec{\beta}_{m z}, & \left|p_{m}(t)\right| \leq \chi_{m}, \\
\overleftarrow{\beta}_{m z}, & \left|p_{m}(t)\right|>\chi_{m},\end{cases}
\end{aligned}
$$

105

where $\vec{\alpha}_{m z}, \overleftarrow{\alpha}_{m z}, \vec{\beta}_{m z}$ and $\overleftarrow{\beta}_{m z}$ are constants, $\chi_{m}>0$ is the switching jump. We denote $\hat{\alpha}_{m z}=\max \left\{\left|\vec{\alpha}_{m z}\right|,\left|\overleftarrow{\alpha}_{m z}\right|\right\}, \hat{\beta}_{m z}=\max \left\{\left|\vec{\beta}_{m z}\right|,\left|\overleftarrow{\beta}_{m z}\right|\right\}, \hat{\Delta}=\left[\hat{\alpha}_{m z}\right]_{l \times l}$, $\hat{\Omega}=\left[\hat{\beta}_{m z}\right]_{l \times l}$. 
Let $q_{m}(t)=\frac{d p_{m}(t)}{d t}+\omega_{m} p_{m}(t), \quad m=1,2, \ldots, l$, where $\omega_{m}$ is a constant, then system (1) can be rewritten as

$$
\left\{\begin{array}{l}
\frac{d p_{m}(t)}{d t}=-\omega_{m} p_{m}(t)+q_{m}(t), \quad m=1,2, \ldots, l \\
\frac{d q_{m}(t)}{d t}=-\left(b_{m}-\omega_{m}\right) q_{m}(t)-\left[c_{m}+\omega_{m}\left(\omega_{m}-b_{m}\right)\right] \\
\times p_{m}(t)+\sum_{z=1}^{l} \alpha_{m z}\left(p_{m}(t)\right) f_{z}\left(p_{z}(t)\right) \\
+\sum_{z=1}^{l} \beta_{m z}\left(p_{m}(t)\right) f_{z}\left(p_{z}\left(t-\tau_{m z}(t)\right)\right)+I_{m}(t) \\
\triangleq-\tilde{b}_{m} q_{m}(t)-\tilde{c}_{m} p_{m}(t)+\sum_{z=1}^{l} \alpha_{m z}\left(p_{m}(t)\right) f_{z}\left(p_{z}(t)\right) \\
+\sum_{z=1}^{l} \beta_{m z}\left(p_{m}(t)\right) f_{z}\left(p_{z}\left(t-\tau_{m z}(t)\right)\right)+I_{m}(t)
\end{array}\right.
$$

where $\tilde{b}_{m}=b_{m}-\omega_{m}, \tilde{c}_{m}=c_{m}+\omega_{m}\left(\omega_{m}-b_{m}\right)$ and the initial conditions are

$$
\left\{\begin{array}{l}
p_{m}(s)=\Upsilon_{m}(s), \\
q_{m}(s)=\Theta_{m}(s)+\omega_{m} \Upsilon_{m}(s), \quad-\tau \leq s \leq 0
\end{array}\right.
$$

Let system (1) or (2) be the drive IMNNs, then the response system can be described in two forms, namely, Form (A) and Form (B).

Form (A):

$$
\left\{\begin{array}{l}
\frac{d \tilde{p}_{m}(t)}{d t}=-\omega_{m} \tilde{p}_{m}(t)+\tilde{q}_{m}(t)+u_{m}(t) \\
\frac{d \tilde{q}_{m}(t)}{d t}=-\tilde{b}_{m} \tilde{q}_{m}(t)-\tilde{c}_{m} \tilde{p}_{m}(t)+\sum_{z=1}^{l} \alpha_{m z}\left(\tilde{p}_{m}(t)\right) \\
\times f_{z}\left(\tilde{p}_{z}(t)\right)+\sum_{z=1}^{l} \beta_{m z}\left(\tilde{p}_{m}(t)\right) f_{z}\left(\tilde{p}_{z}\left(t-\tau_{m z}(t)\right)\right) \\
+I_{m}(t)+v_{m}(t)
\end{array}\right.
$$

where $u_{m}(t)$ and $v_{m}(t)$ are controllers.

Form (B):

$$
\begin{aligned}
& \frac{d^{2} \tilde{p}_{m}(t)}{d t^{2}}=-b_{m} \frac{d \tilde{p}_{m}(t)}{d t}-c_{m} \tilde{p}_{m}(t)+\sum_{z=1}^{l} \alpha_{m z}\left(\tilde{p}_{m}(t)\right) \\
& \times f_{z}\left(\tilde{p}_{z}(t)\right)+\sum_{z=1}^{l} \beta_{m z}\left(\tilde{p}_{m}(t)\right) f_{z}\left(\tilde{p}_{z}\left(t-\tau_{m z}(t)\right)\right) \\
& +I_{m}(t)+v_{m}(t), m=1,2, \ldots, l,
\end{aligned}
$$

where $v_{m}(t)$ represents the controller. 
Let $\tilde{q}_{m}(t)=\frac{d \tilde{p}_{m}(t)}{d t}+\omega_{m} \tilde{p}_{m}(t), \quad m=1,2, \ldots, l$, where $\omega_{m}$ is a constant. Then system (4) can be rewritten as

$$
\left\{\begin{array}{l}
\frac{d \tilde{p}_{m}(t)}{d t}=-\omega_{m} \tilde{p}_{m}(t)+\tilde{q}_{m}(t) \\
\frac{d \tilde{q}_{m}(t)}{d t}=-\tilde{b}_{m} \tilde{q}_{m}(t)-\tilde{c}_{m} \tilde{p}_{m}(t)+\sum_{z=1}^{l} \alpha_{m z}\left(\tilde{p}_{m}(t)\right) \\
\times f_{z}\left(\tilde{p}_{z}(t)\right)+\sum_{z=1}^{l} \beta_{m z}\left(\tilde{p}_{m}(t)\right) f_{z}\left(\tilde{p}_{z}\left(t-\tau_{m z}(t)\right)\right) \\
+I_{m}(t)+v_{m}(t)
\end{array}\right.
$$

where $\tilde{b}_{m}=b_{m}-\omega_{m}, \tilde{c}_{m}=c_{m}+\omega_{m}\left(\omega_{m}-b_{m}\right)$

We define errors $e_{m}(t)=\tilde{p}_{m}(t)-p_{m}(t)$ and $r_{m}(t)=\tilde{q}_{m}(t)-q_{m}(t)$.

When the response system is system (3), we can get the errors as

$$
\left\{\begin{array}{l}
\frac{d e_{m}(t)}{d t}=-\omega_{m} e_{m}(t)+r_{m}(t)+u_{m}(t) \\
\frac{d r_{m}(t)}{d t}=-\tilde{b}_{m} r_{m}(t)-\tilde{c}_{m} e_{m}(t) \\
+\sum_{z=1}^{l} \alpha_{m z}\left(\tilde{p}_{m}(t)\right) g_{z}\left(e_{z}(t)\right) \\
+\sum_{z=1}^{l}\left(\alpha_{m z}\left(\tilde{p}_{m}(t)\right)-\alpha_{m z}\left(p_{m}(t)\right)\right) f_{z}\left(p_{z}(t)\right) \\
+\sum_{z=1}^{l} \beta_{m z}\left(\tilde{p}_{m}(t)\right) g_{z}\left(e_{z}\left(t-\tau_{m z}(t)\right)\right) \\
+\sum_{z=1}^{l}\left(\beta_{m z}\left(\tilde{p}_{m}(t)\right)-\beta_{m z}\left(p_{m}(t)\right)\right) \\
\times f_{z}\left(p_{z}\left(t-\tau_{m z}(t)\right)\right)+v_{m}(t)
\end{array}\right.
$$

where $g_{z}\left(e_{z}(t)\right)=f_{z}\left(\tilde{p}_{z}(t)\right)-f_{z}\left(p_{z}(t)\right)$. Moreover, the vector form of system (6) can be written as

$$
\left\{\begin{array}{l}
\frac{d e(t)}{d t}=-W e(t)+r(t)+u(t) \\
\frac{d r(t)}{d t}=-\tilde{B} r(t)-\tilde{C} e(t)+\Delta(\tilde{p}(t)) g(e(t)) \\
+(\Delta(\tilde{p}(t))-\Delta(p(t))) f(p(t)) \\
+\Omega(\tilde{p}(t)) g(e(t-\tau(t))) \\
+(\Omega(\tilde{p}(t))-\Omega(p(t))) f(p(t-\tau(t)))+v(t) .
\end{array}\right.
$$

where $e(t)=\left(e_{1}(t), e_{2}(t), \ldots, e_{l}(t)\right)^{T}, r(t)=\left(r_{1}(t), r_{2}(t), \ldots, r_{l}(t)\right)^{T}, W=$ $\operatorname{diag}\left\{\omega_{1}, \omega_{2}, \ldots, \omega_{l}\right\}, u(t)=\left(u_{1}(t), u_{2}(t), \ldots, u_{l}(t)\right)^{T}, \tilde{B}=\operatorname{diag}\left\{\tilde{b}_{1}, \tilde{b}_{2}, \ldots, \tilde{b}_{l}\right\}$, $\tilde{C}=\operatorname{diag}\left\{\tilde{c}_{1}, \tilde{c}_{2}, \ldots, \tilde{c}_{l}\right\}, g(e(t))=\left(g_{1}\left(e_{1}(t)\right), g_{2}\left(e_{2}(t)\right), \ldots, g_{l}\left(e_{l}(t)\right)\right)^{T}, f(p(t))=$ 
$\left(f_{1}\left(p_{1}(t)\right), f_{2}\left(p_{2}(t)\right), \ldots, f_{l}\left(p_{l}(t)\right)\right)^{T}, \Delta(\tilde{p}(t))=\left[\alpha_{m z}(\tilde{p}(t))\right]_{l \times l}, \Delta(p(t))=\left[\alpha_{m z}(p(t))\right]_{l \times l}$, $\Omega(\tilde{p}(t))=\left[\beta_{m z}(\tilde{p}(t))\right]_{l \times l}, \Omega(p(t))=\left[\beta_{m z}(p(t))\right]_{l \times l}, v(t)=\left(v_{1}(t), v_{2}(t), \ldots, v_{l}(t)\right)^{T}$.

When the response system is system (5), we can get the errors as

$$
\left\{\begin{array}{l}
\frac{d e_{m}(t)}{d t}=-\omega_{m} e_{m}(t)+r_{m}(t) \\
\frac{d r_{m}(t)}{d t}=-\tilde{b}_{m} r_{m}(t)-\tilde{c}_{m} e_{m}(t) \\
+\sum_{z=1}^{l} \alpha_{m z}\left(\tilde{p}_{m}(t)\right) g_{z}\left(e_{z}(t)\right) \\
+\sum_{z=1}^{l}\left(\alpha_{m z}\left(\tilde{p}_{m}(t)\right)-\alpha_{m z}\left(p_{m}(t)\right)\right) f_{z}\left(p_{z}(t)\right) \\
+\sum_{z=1}^{l} \beta_{m z}\left(\tilde{p}_{m}(t)\right) g_{z}\left(e_{z}\left(t-\tau_{m z}(t)\right)\right) \\
+\sum_{z=1}^{l}\left(\beta_{m z}\left(\tilde{p}_{m}(t)\right)-\beta_{m z}\left(p_{m}(t)\right)\right) \\
\times f_{z}\left(p_{z}\left(t-\tau_{m z}(t)\right)\right)+v_{m}(t),
\end{array}\right.
$$

and the vector form

$$
\left\{\begin{array}{l}
\frac{d e(t)}{d t}=-W e(t)+r(t), \\
\frac{d r(t)}{d t}=-\tilde{B} r(t)-\tilde{C} e(t)+\Delta(\tilde{p}(t)) g(e(t)) \\
+(\Delta(\tilde{p}(t))-\Delta(p(t))) f(p(t)) \\
+\Omega(\tilde{p}(t)) g(e(t-\tau(t))) \\
+(\Omega(\tilde{p}(t))-\Omega(p(t))) f(p(t-\tau(t)))+v(t),
\end{array}\right.
$$

120

where $v(t)=\left(v_{1}(t), v_{2}(t), \ldots, v_{l}(t)\right)^{T}$.

We define measured errors as $m e(t)=e\left(t_{i}\right)-e(t)$ and $m r(t)=r\left(t_{i}\right)-r(t)$, $\forall t \in\left[t_{i}, t_{i+1}\right)$. In ETC strategy, the state-dependent threshold needs to be set. When the measured errors exceed the threshold, the control will be updated under a new triggering event. It is worth noting that $\lim _{t \rightarrow t_{i}^{+}} m e(t)=m e\left(t_{i}\right)=$ ${ }^{125}$ $0, \lim _{t \rightarrow t_{i}^{+}} m r(t)=m r\left(t_{i}\right)=0, \lim _{t \rightarrow t_{i}^{-}} m e(t)=\lim _{t \rightarrow t_{i}^{-}} e\left(t_{i-1}\right)-e(t) \neq 0$ and $\lim _{t \rightarrow t_{i}^{-}} m r(t)=\lim _{t \rightarrow t_{i}^{-}} r\left(t_{i-1}\right)-r(t) \neq 0$. Therefore, $m e(t)$ and $m r(t)$ are discontinuous at $t=t_{i}$.

Because time-varying delay $\tau_{m z}(t)$ satisfies $0 \leq \tau_{m z}(t) \leq \tau$, and $t \in\left[t_{i}, t_{i+1}\right)$, then we can acquire $t-\tau_{m z}(t) \in\left[t_{i}-\tau, t_{i+1}\right)$, where $\tau$ is a positive constant. It ${ }_{130}$ is worth nothing that the term $t-\tau_{m z}(t)$ does not affect event-triggered control conditions introduced in the Section 3. 
Definition 1. If

$$
\lim _{t \rightarrow \infty}\|\tilde{p}(t)-p(t)\|_{1}=0,
$$

and

$$
\lim _{t \rightarrow \infty}\|\tilde{q}(t)-q(t)\|_{1}=0,
$$

then IMNNs systems (2) and (3) (or (5)) can achieve asymptotical synchronization, where $\tilde{p}(t)=\left(\tilde{p}_{1}(t), \tilde{p}_{2}(t), \ldots, \tilde{p}_{l}(t)\right)^{T}, p(t)=\left(p_{1}(t), p_{2}(t), \ldots, p_{l}(t)\right)^{T}$, $\tilde{q}(t)=\left(\tilde{q}_{1}(t), \tilde{q}_{2}(t), \ldots, \tilde{q}_{l}(t)\right)^{T}, q(t)=\left(q_{1}(t), q_{2}(t), \ldots, q_{l}(t)\right)^{T}$.

135 will discuss two types of controllers in this section. The first type of controller is added to the transformational first-order response system (3), and the second type of controller is added to the original second-order response system (4).

\subsection{The First Type of Controller}

We consider the first type of state feedback controller in the Form (A) as follows

$$
\left\{\begin{array}{l}
u(t)=-\mathrm{H} e\left(t_{i}\right), \\
v(t)=-\Lambda r\left(t_{i}\right)-\Gamma \operatorname{sgn}\left(r\left(t_{i}\right)\right), t \in\left[t_{i}, t_{i+1}\right),
\end{array}\right.
$$

150

where $\mathrm{H}=\operatorname{diag}\left(h_{1}, h_{2}, \ldots, h_{l}\right)^{T}$ and $\Lambda=\operatorname{diag}\left(\Lambda_{1}, \Lambda_{2}, \ldots, \Lambda_{l}\right)^{T}$ are positive definite matrices; $\Gamma=\operatorname{diag}\left(\Gamma_{1}, \Gamma_{2}, \ldots, \Gamma_{l}\right)^{T} ; \operatorname{sgn}()$ represents sign function; and $t_{i}$ is a release instant. 
Assumption 1. Time-varying delay $\tau_{m z}(t)$ satisfies

$$
\dot{\tau}_{m z}(t) \leq \theta<1
$$

where $\theta$ is a positive constant.

Assumption 2. Activation function $f_{z}$ is bounded and satisfies Lipschitz

155 condition, namely, $\left|f_{z}\left(U_{1}\right)\right| \leq N_{z}$ and $\left|f_{z}\left(U_{1}\right)-f_{z}\left(U_{2}\right)\right| \leq M_{z}\left|U_{1}-U_{2}\right|$ for any $U_{1}, U_{2} \in \Re$, where $M_{z}>0$ and $N_{z}>0$ are constants, $z=1,2, \ldots, l$.

\section{(1) Static Event-Triggered Control}

Theorem 1. IMNNs systems (2) and (3) can be synchronized under assumptions 1 and 2 with the first type of state feedback controller (10) and the following SETC conditions

$$
\begin{gathered}
\|m e(t)\|_{1} \leq \xi_{1} \frac{\mu_{1}\|e(t)\|_{1}}{\max \{\lambda(\mathrm{H})\}}, \\
\|m r(t)\|_{1} \leq \xi_{2} \frac{\left(\mu_{2}\|r(t)\|_{1}+\varpi\right)}{\max \{\lambda(\Lambda)\}},
\end{gathered}
$$

for $t \in\left[t_{i}, t_{i+1}\right)$, if

$$
\begin{gathered}
\min \{\lambda(\mathrm{H})\}>-\min \{\lambda(W)\}+\max \{|\lambda(\tilde{C})|\} \\
+\frac{M_{\max }}{1-\theta}\|\hat{\Omega}\|_{1}+M_{\max }\|\hat{\Delta}\|_{1} \\
\min \{\lambda(\Lambda)\}>1-\min \{\lambda(\tilde{B})\} \\
\left\{\begin{array}{l}
\Gamma_{m}>\kappa_{m}, \quad \text { if } \operatorname{sgn}\left(r_{m}(t)\right) \operatorname{sgn}\left(r_{m}\left(t_{i}\right)\right)>0 \\
\Gamma_{m} \leq-\kappa_{m}, \quad \text { otherwise }
\end{array}\right.
\end{gathered}
$$

and

$$
\kappa_{m}>\sum_{z=1}^{l}\left[\left|\vec{\alpha}_{m z}-\overleftarrow{\alpha}_{m z}\right|+\left|\vec{\beta}_{m z}-\overleftarrow{\beta}_{m z}\right|\right] N_{z}
$$

where $\xi_{1}, \xi_{2} \in[0,1], M_{\max }=\max _{1 \leq z \leq l}\left\{M_{z}\right\}$,

$$
\begin{aligned}
\mu_{1}= & \min \{\lambda(W)\}-\max \{|\lambda(\tilde{C})|\}-\frac{M_{\max }}{1-\theta}\|\hat{\Omega}\|_{1} \\
& -M_{\max }\|\hat{\Delta}\|_{1}+\min \{\lambda(\mathrm{H})\},
\end{aligned}
$$




$$
\mu_{2}=-1+\min \{\lambda(\tilde{B})\}+\min \{\lambda(\Lambda)\},
$$

and

$$
\varpi=\sum_{m=1}^{l}\left\{\kappa_{m}-\sum_{z=1}^{l}\left[\left|\vec{\alpha}_{m z}-\overleftarrow{\alpha}_{m z}\right|+\left|\vec{\beta}_{m z}-\overleftarrow{\beta}_{m z}\right|\right] N_{z}\right\} .
$$

Proof. Consider a Lyapunov function as

$$
\begin{aligned}
& V(t)=\|e(t)\|_{1}+\|r(t)\|_{1} \\
& +\sum_{m=1}^{l} \sum_{z=1}^{l} \frac{\hat{\beta}_{m z}}{1-\theta} \int_{t-\tau_{m z}(t)}^{t}\left|g_{z}\left(e_{z}(s)\right)\right| d s
\end{aligned}
$$

For $t \in\left[t_{i}, t_{i+1}\right)$, we can get the upper right Dini-derivative of $V(t)$ as

$$
\begin{aligned}
& \dot{V}(t) \leq \operatorname{sgn}^{T}(e(t)) \dot{e}(t)+\operatorname{sgn}^{T}(r(t)) \dot{r}(t) \\
& +\sum_{m=1}^{l} \sum_{z=1}^{l} \hat{\beta}_{m z}\left[\frac{1}{1-\theta}\left|g_{z}\left(e_{z}(t)\right)\right|-\left|g_{z}\left(e_{z}\left(t-\tau_{m z}(t)\right)\right)\right|\right] \\
& =\operatorname{sgn}^{T}(e(t))\left\{-W e(t)+r(t)-\mathrm{He}\left(t_{i}\right)\right\} \\
& +\operatorname{sgn}^{T}(r(t))\{-\tilde{B} r(t)-\tilde{C} e(t)+\Delta(\tilde{p}(t)) g(e(t)) \\
& +(\Delta(\tilde{p}(t))-\Delta(p(t))) f(p(t)) \\
& +\Omega(\tilde{p}(t)) g(e(t-\tau(t)))+(\Omega(\tilde{p}(t))-\Omega(p(t))) \\
& \left.\times f(p(t-\tau(t)))-\Lambda r\left(t_{i}\right)-\Gamma \operatorname{sgn}\left(r\left(t_{i}\right)\right)\right\} \\
& +\sum_{m=1}^{l} \sum_{z=1}^{l} \hat{\beta}_{m z}\left[\frac{1}{1-\theta}\left|g_{z}\left(e_{z}(t)\right)\right|-\left|g_{z}\left(e_{z}\left(t-\tau_{m z}(t)\right)\right)\right|\right] \\
& \leq-\min \{\lambda(W)\}\|e(t)\|_{1}+\|r(t)\|_{1}-\operatorname{sgn}^{T}(e(t)) \mathrm{He}\left(t_{i}\right) \\
& -\min \{\lambda(\tilde{B})\}\|r(t)\|_{1}+\max \{|\lambda(\tilde{C})|\}\|e(t)\|_{1} \\
& +M_{\max }\|\hat{\Delta}\|_{1}\|e(t)\|_{1}+\operatorname{sgn}^{T}(r(t))\{(\Delta(\tilde{p}(t))-\Delta(p(t))) \\
& \times f(p(t))+(\Omega(\tilde{p}(t))-\Omega(p(t))) f(p(t-\tau(t))) \\
& \left.-\Lambda r\left(t_{i}\right)-\Gamma \operatorname{sgn}\left(r\left(t_{i}\right)\right)\right\}+\frac{M_{\max }}{1-\theta}\|\hat{\Omega}\|_{1}\|e(t)\|_{1}
\end{aligned}
$$

Combining with $m e(t)=e\left(t_{i}\right)-e(t)$ and $m r(t)=r\left(t_{i}\right)-r(t)$, we get

$$
\begin{aligned}
& -\operatorname{sgn}^{T}(e(t)) \mathrm{H} e\left(t_{i}\right)=-\operatorname{sgn}^{T}(e(t)) \mathrm{H}(e(t)+m e(t)) \\
& \leq-\min \{\lambda(\mathrm{H})\}\|e(t)\|_{1}+\max \{\lambda(\mathrm{H})\}\|m e(t)\|_{1}, \\
& -\operatorname{sgn}^{T}(r(t)) \Lambda r\left(t_{i}\right)=-\operatorname{sgn}^{T}(r(t)) \Lambda(r(t)+m r(t)) \\
& \leq-\min \{\lambda(\Lambda)\}\|r(t)\|_{1}+\max \{\lambda(\Lambda)\}\|m r(t)\|_{1} .
\end{aligned}
$$


According to Assumption 2, the following inequalities hold.

$$
\begin{aligned}
& \operatorname{sgn}^{T}(r(t))\{(\Delta(\tilde{p}(t))-\Delta(p(t))) f(p(t)) \\
& \left.+(\Omega(\tilde{p}(t))-\Omega(p(t))) f(p(t-\tau(t)))-\Gamma \operatorname{sgn}\left(r\left(t_{i}\right)\right)\right\} \\
& \leq \sum_{m=1}^{l} \sum_{z=1}^{l}\left[\left|\vec{\alpha}_{m z}-\overleftarrow{\alpha}_{m z}\right|+\left|\vec{\beta}_{m z}-\overleftarrow{\beta}_{m z}\right|\right] N_{z} \\
& -\sum_{m=1}^{l} \operatorname{sgn}\left(r_{m}(t)\right) \operatorname{sgn}\left(r_{m}\left(t_{i}\right)\right) \Gamma_{m} \\
& \leq-\sum_{m=1}^{l}\left\{\kappa_{m}-\sum_{z=1}^{l}\left[\left|\vec{\alpha}_{m z}-\overleftarrow{\alpha}_{m z}\right|+\left|\vec{\beta}_{m z}-\overleftarrow{\beta}_{m z}\right|\right] N_{z}\right\} \\
& =-\varpi<0
\end{aligned}
$$

Then, we can get that

$$
\begin{aligned}
& \dot{V}(t) \leq\left[-\min \{\lambda(W)\}+\max \{|\lambda(\tilde{C})|\}+\frac{M_{\max }}{1-\theta}\|\hat{\Omega}\|_{1}\right. \\
& \left.+M_{\max }\|\hat{\Delta}\|_{1}-\min \{\lambda(\mathrm{H})\}\right]\|e(t)\|_{1}+\max \{\lambda(\mathrm{H})\} \\
& \times\|\operatorname{me}(t)\|_{1}+[1-\min \{\lambda(\tilde{B})\}-\min \{\lambda(\Lambda)\}] \\
& \times\|r(t)\|_{1}+\max \{\lambda(\Lambda)\}\|m r(t)\|_{1}-\varpi \\
& \leq\left(\xi_{1}-1\right) \mu_{1}\|e(t)\|_{1}+\left(\xi_{2}-1\right)\left(\mu_{2}\|r(t)\|_{1}+\varpi\right) \\
& \leq 0
\end{aligned}
$$

Thus, the system (3) can achieve synchronization with the system (2) under the SETC conditions (11) and (12). The proof is finished.

The type of synchronization achieved in this paper is asymptotical synchronization. The derivative of Lyapunov function $V(t)$ is not more than 0 , and each term of Lyapunov function $V(t)$ in (17) is nonnegative. Thus, $\|e(t)\|_{1}$ and $\|r(t)\|_{1}$ are asymptotically stable as time goes on, in other words,

$$
\lim _{t \rightarrow \infty}\|\tilde{p}(t)-p(t)\|_{1}=0
$$

and

$$
\lim _{t \rightarrow \infty}\|\tilde{q}(t)-q(t)\|_{1}=0 .
$$

This means the synchronization between system (3) and system (2) is asymptotical according to the Definition 1. Similarly, the synchronization between system (3) and system (2) in the following theorems and corollaries via SETC or DETC is also asymptotical. 
The feedback controller (10) is an essential item of realizing synchronization

165 applied in errors $e(t)$ and $r(t)$ to make error systems stable. In addition, $u(t)$ and $v(t)$ are unchanged when time $t \in\left[t_{i}, t_{i+1}\right)$, which means that computing cost decreases due to reducing the update times of feedback controller (10).

Remark 2. When SETC conditions (11) and (12) are violated, the controller re-do the calculation as long as SETC conditions (11) and (12) are satisfied. Therefore, compared with the traditional continuous-time control methods [3237], ETC scheme can reduce communication burden based on ensuring system performance.

Remark 3. Due to the limited communication resources and channel bandwidth, it is very necessary to reduce the update times of controller and data transmission rate. ETC scheme for the synchronization of IMNNs can effectively reduce the update times of controller and decrease computing cost. Therefore, ETC scheme for IMNNs is very practical and meaningful. been studied. These papers focused on the first order derivative of the state variables and considered one type of error variable, that is to say, one type of measured error was addressed to achieve synchronization. Compared with the first order derivative of the state variables, the second order derivative is more complicated and harder to be addressed. Currently, the solution for the second order derivative is to transform the second-order system into two first-order systems [28-37], which means it needs to consider two types of error variables, namely two types of measured errors. Therefore, the one error variable strategy in [46-48] cannot solve the synchronization problem of IMNN systems [28-37]. the synchronization of IMNNs with time-varying delays via new ETC condition and state feedback controller for the first time.

Corollary 1. IMNNs systems (2) and (3) can be synchronized under as- 
sumptions 1 and 2 with the first type of state feedback controller (10) and the following SETC conditions

$$
\begin{gathered}
\|m e(t)\|_{1} \leq \xi_{1} \frac{\left(\mu_{1}\|e(t)\|_{1}+\varpi\right)}{\max \{\lambda(\mathrm{H})\}}, \\
\|m r(t)\|_{1} \leq \xi_{2} \frac{\mu_{2}\|r(t)\|_{1}}{\max \{\lambda(\Lambda)\}}
\end{gathered}
$$

for $t \in\left[t_{i}, t_{i+1}\right)$, if matrices $\mathrm{H}, \Lambda$ and $\Gamma$ satisfy (13)-(16), and $\xi_{1}, \xi_{2}, \mu_{1}, \mu_{2}$ and $\varpi$ are the same as Theorem 1 .

Proof. Combining the proof of Theorem 1 with (18) and (19), we get

$$
\begin{aligned}
& \dot{V}(t) \leq\left[-\min \{\lambda(W)\}+\max \{|\lambda(\tilde{C})|\}+\frac{M_{\max }}{1-\theta}\|\hat{\Omega}\|_{1}\right. \\
& \left.+M_{\max }\|\hat{\Delta}\|_{1}-\min \{\lambda(\mathrm{H})\}\right]\|e(t)\|_{1}+\max \{\lambda(\mathrm{H})\} \\
& \times\|\operatorname{me}(t)\|_{1}+[1-\min \{\lambda(\tilde{B})\}-\min \{\lambda(\Lambda)\}] \\
& \times\|r(t)\|_{1}+\max \{\lambda(\Lambda)\}\|m r(t)\|_{1}-\varpi \\
& \leq\left(\xi_{1}-1\right)\left(\mu_{1}\|e(t)\|_{1}+\varpi\right)+\left(\xi_{2}-1\right) \mu_{2}\|r(t)\|_{1} \\
& \leq 0
\end{aligned}
$$

Thus, the system (3) can achieve synchronization with the system (2) under the SETC conditions (18) and (19). The proof is finished.

Corollary 2. IMNNs systems (2) and (3) can be synchronized under assumptions 1 and 2 with the first type of state feedback controller (10) and the following SETC conditions

$$
\begin{gathered}
\|m e(t)\|_{1} \leq \frac{\xi_{1} \mu_{1}\|e(t)\|_{1}+\ell \varpi}{\max \{\lambda(\mathrm{H})\}}, \\
\|m r(t)\|_{1} \leq \frac{\xi_{2} \mu_{2}\|r(t)\|_{1}+(1-\ell) \varpi}{\max \{\lambda(\Lambda)\}}
\end{gathered}
$$

for $t \in\left[t_{i}, t_{i+1}\right), \ell \in(0,1)$, if matrices $\mathrm{H}, \Lambda$ and $\Gamma$ satisfy (13)-(16), and $\xi_{1}, \xi_{2}$, $\mu_{1}, \mu_{2}$ and $\varpi$ are the same as Theorem 1.

Corollary 3. IMNNs systems (2) and (3) can be synchronized under assumptions 1 and 2 with the first type of state feedback controller (10) and the following SETC conditions

$$
\|m e(t)\|_{1} \leq \frac{\xi_{1} \mu_{1}\left\|e\left(t_{i}\right)\right\|_{1}+\ell \varpi}{\max \{\lambda(\mathrm{H})\}+\xi_{1} \mu_{1}},
$$




$$
\|m r(t)\|_{1} \leq \frac{\xi_{2} \mu_{2}\left\|r\left(t_{i}\right)\right\|_{1}+(1-\ell) \varpi}{\max \{\lambda(\Lambda)\}+\xi_{2} \mu_{2}},
$$
$\mu_{1}, \mu_{2}$ and $\varpi$ are the same as Theorem 1 .

Proof. From (22) and (23), we can get

$$
\begin{gathered}
\max \{\lambda(\mathrm{H})\}\|m e(t)\|_{1} \leq \xi_{1} \mu_{1}\left(\left\|e\left(t_{i}\right)\right\|_{1}-\|m e(t)\|_{1}\right)+\ell \varpi \\
\leq \xi_{1} \mu_{1}\|e(t)\|_{1}+\ell \varpi, \\
\quad \max \{\lambda(\Lambda)\}\|m r(t)\|_{1} \\
\leq \xi_{2} \mu_{2}\left(\left\|r\left(t_{i}\right)\right\|_{1}-\|m r(t)\|_{1}\right)+(1-\ell) \varpi \\
\leq \xi_{2} \mu_{2}\|r(t)\|_{1}+(1-\ell) \varpi,
\end{gathered}
$$

for $t \in\left[t_{i}, t_{i+1}\right)$, in other words, the inequalities (20) and (21) in Corollary 2 hold. Thus, all the conditions of Corollary 2 are satisfied.

Remark 5. Because the state feedback controller (10) is added to the transformational first-order system (3), it is very convenient to set ETC conditions. From Theorem 1 and corollaries 1-3, we have lots of choices to set the condition$\mathrm{s}$ of measured errors $m e(t)$ and $m r(t)$, which the existing methods for MNNs introduced in [46-48] cannot meet. Therefore, the first type of state feedback controller (10) is very necessary and flexible for studying the synchronization of IMNNs and choosing ETC conditions.

\section{(2) Dynamic Event-Triggered Control}

We set two dynamic variables $\sigma_{1}(t)$ and $\sigma_{2}(t)$, which satisfy the following conditions

$$
\begin{gathered}
\dot{\sigma}_{1}(t)=-\sigma_{1}(t)+\xi_{1} \mu_{1}\|e(t)\|_{1} \\
-\max \{\lambda(\mathrm{H})\}\|m e(t)\|_{1}, \\
\dot{\sigma}_{2}(t)=-\sigma_{2}(t)+\xi_{2}\left(\mu_{2}\|r(t)\|_{1}+\varpi\right) \\
-\max \{\lambda(\Lambda)\}\|m r(t)\|_{1},
\end{gathered}
$$

where matrices $\mathrm{H}, \Lambda$ satisfy (13)-(14), and $\xi_{1}, \xi_{2}, \mu_{1}, \mu_{2}$ and $\varpi$ are the same as Theorem 1. The initial values of (24) and (25) are $\sigma_{1}(0)$ and $\sigma_{2}(0)$, and satisfy $\sigma_{1}(0) \geq 0$ and $\sigma_{2}(0) \geq 0$. 
The dynamic variables $\sigma_{1}(t)$ and $\sigma_{2}(t)$ are used to control the thresholds of measured errors $m e(t)$ and $m r(t)$ in DETC scheme. For example, error $e(t)$ and dynamic variable $\sigma_{1}(t)$ are applied to dynamically decide the threshold of measured errors me(t) in (26); error $r(t)$ and dynamic variable $\sigma_{2}(t)$ are applied to dynamically decide the threshold of measured errors $m r(t)$ in $(27)$. When the measured errors $m e(t)$ and $m r(t)$ exceed the thresholds provided in (26) and (27), the control will be updated under a new triggering event. By introducing dynamic variables, the update times of controller under DETC scheme is less than that under SETC scheme.

Theorem 2. IMNNs systems (2) and (3) can be synchronized under assumptions 1 and 2 with the first type of state feedback controller (10) and the following DETC conditions

$$
\begin{gathered}
\|m e(t)\|_{1} \leq \sigma_{1}(t)+\xi_{1} \frac{\mu_{1}\|e(t)\|_{1}}{\max \{\lambda(\mathrm{H})\}}, \\
\|m r(t)\|_{1} \leq \sigma_{2}(t)+\xi_{2} \frac{\left(\mu_{2}\|r(t)\|_{1}+\varpi\right)}{\max \{\lambda(\Lambda)\}},
\end{gathered}
$$

for $t \in\left[t_{i}, t_{i+1}\right)$, if matrices $\mathrm{H}, \Lambda$ and $\Gamma$ satisfy (13)-(16), and $\xi_{1}, \xi_{2}, \mu_{1}, \mu_{2}$ and $225 \varpi$ are the same as Theorem 1 .

Proof. From (24)-(27), we can get

$$
\begin{aligned}
& \dot{\sigma}_{1}(t) \geq-\sigma_{1}(t)+\max \{\lambda(\mathrm{H})\}\left(\|m e(t)\|_{1}-\sigma_{1}(t)\right) \\
& -\max \{\lambda(\mathrm{H})\}\|m e(t)\|_{1}=-(1+\max \{\lambda(\mathrm{H})\}) \sigma_{1}(t),
\end{aligned}
$$

and

$$
\begin{aligned}
& \dot{\sigma}_{2}(t) \geq-\sigma_{2}(t)+\max \{\lambda(\Lambda)\}\left(\|m r(t)\|_{1}-\sigma_{2}(t)\right) \\
& -\max \{\lambda(\Lambda)\}\|m r(t)\|_{1}=-(1+\max \{\lambda(\Lambda)\}) \sigma_{2}(t),
\end{aligned}
$$

Therefore, we can obtain that $\sigma_{1}(t) \geq 0$ and $\sigma_{2}(t) \geq 0$ according to Lemma 1.

We define the following function

$$
V_{1}(t)=V(t)+\sigma_{1}(t)+\sigma_{2}(t)
$$

where $V(t)$ is the same as (17). 
For $t \in\left[t_{i}, t_{i+1}\right)$, we can get the upper right Dini-derivative of $V_{1}(t)$ as

$$
\begin{aligned}
& \dot{V}_{1}(t)=\dot{V}(t)+\dot{\sigma}_{1}(t)+\dot{\sigma}_{2}(t) \\
& \leq\left[-\min \{\lambda(W)\}+\max \{|\lambda(\tilde{C})|\}+\frac{M_{\max }}{1-\theta}\|\hat{\Omega}\|_{1}\right. \\
& \left.+M_{\max }\|\hat{\Delta}\|_{1}-\min \{\lambda(\mathrm{H})\}\right]\|e(t)\|_{1} \\
& +\max \{\lambda(\mathrm{H})\}\|m e(t)\|_{1}+[1-\min \{\lambda(\tilde{B})\}-\min \{\lambda(\Lambda)\}] \\
& \times\|r(t)\|_{1}+\max \{\lambda(\Lambda)\}\|m r(t)\|_{1}-\varpi \\
& -\sigma_{1}(t)+\xi_{1} \mu_{1}\|e(t)\|_{1}-\max \{\lambda(\mathrm{H})\}\|m e(t)\|_{1} \\
& -\sigma_{2}(t)+\xi_{2}\left(\mu_{2}\|r(t)\|_{1}+\varpi\right)-\max \{\lambda(\Lambda)\}\|m r(t)\|_{1} \\
& =-\mu_{1}\|e(t)\|_{1}-\sigma_{1}(t)+\xi_{1} \mu_{1}\|e(t)\|_{1}-\mu_{2}\|r(t)\|_{1} \\
& -\varpi-\sigma_{2}(t)+\xi_{2}\left(\mu_{2}\|r(t)\|_{1}+\varpi\right) \\
& \leq\left(\xi_{1}-1\right) \mu_{1}\|e(t)\|_{1}+\left(\xi_{2}-1\right)\left(\mu_{2}\|r(t)\|_{1}+\varpi\right) \\
& -\sigma_{1}(t)-\sigma_{2}(t) \\
& \leq 0
\end{aligned}
$$

Therefore, IMNNs systems (2) and (3) can be synchronized with the state finished.

Similar to Theorem 2, we can have the following corollaries.

We introduce two dynamic variables $\sigma_{3}(t)$ and $\sigma_{4}(t)$, which satisfy the following conditions

$$
\begin{gathered}
\dot{\sigma}_{3}(t)=-\sigma_{3}(t)+\xi_{1}\left(\mu_{1}\|e(t)\|_{1}+\varpi\right) \\
-\max \{\lambda(\mathrm{H})\}\|m e(t)\|_{1}, \\
\dot{\sigma}_{4}(t)=-\sigma_{4}(t)+\xi_{2} \mu_{2}\|r(t)\|_{1}-\max \{\lambda(\Lambda)\}\|m r(t)\|_{1},
\end{gathered}
$$

where matrices H, $\Lambda$ satisfy (13)-(14), and $\xi_{1}, \xi_{2}, \mu_{1}, \mu_{2}$ and $\varpi$ are the same as Theorem 1. The initial values are $\sigma_{3}(0)$ and $\sigma_{4}(0)$, and satisfy $\sigma_{3}(0) \geq 0$ and ${ }_{235} \sigma_{4}(0) \geq 0$.

Corollary 4. IMNNs systems (2) and (3) can be synchronized under assumptions 1 and 2 with the first type of state feedback controller (10) and the following DETC conditions

$$
\|m e(t)\|_{1} \leq \sigma_{3}(t)+\xi_{1} \frac{\left(\mu_{1}\|e(t)\|_{1}+\varpi\right)}{\max \{\lambda(\mathrm{H})\}},
$$




$$
\|m r(t)\|_{1} \leq \sigma_{4}(t)+\xi_{2} \frac{\mu_{2}\|r(t)\|_{1}}{\max \{\lambda(\Lambda)\}},
$$

for $t \in\left[t_{i}, t_{i+1}\right)$, if matrices $\mathrm{H}, \Lambda$ and $\Gamma$ satisfy (13)-(16), and $\xi_{1}, \xi_{2}, \mu_{1}, \mu_{2}$ and $\varpi$ are the same as Theorem 1.

Similarly, we denote two dynamic variables $\sigma_{5}(t)$ and $\sigma_{6}(t)$, which satisfy the following conditions

$$
\begin{gathered}
\dot{\sigma}_{5}(t)=-\sigma_{5}(t)+\xi_{1} \mu_{1}\|e(t)\|_{1}+\ell \varpi \\
-\max \{\lambda(\mathrm{H})\}\|m e(t)\|_{1}, \\
\dot{\sigma}_{6}(t)=-\sigma_{6}(t)+\xi_{2} \mu_{2}\|r(t)\|_{1}+(1-\ell) \varpi \\
-\max \{\lambda(\Lambda)\}\|m r(t)\|_{1},
\end{gathered}
$$

where matrices $\mathrm{H}, \Lambda$ satisfy (13)-(14), $\ell \in(0,1)$, and $\xi_{1}, \xi_{2}, \mu_{1}, \mu_{2}$ and $\varpi$ are the same as Theorem 1. The initial values are $\sigma_{5}(0)$ and $\sigma_{6}(0)$, and satisfy $\sigma_{5}(0) \geq 0$ and $\sigma_{6}(0) \geq 0$.

Corollary 5. IMNNs systems (2) and (3) can be synchronized under assumptions 1 and 2 with the first type of state feedback controller (10) and the following DETC conditions

$$
\begin{gathered}
\|m e(t)\|_{1} \leq \sigma_{5}(t)+\frac{\xi_{1} \mu_{1}\|e(t)\|_{1}+\ell \varpi}{\max \{\lambda(\mathrm{H})\}}, \\
\|m r(t)\|_{1} \leq \sigma_{6}(t)+\frac{\xi_{2} \mu_{2}\|r(t)\|_{1}+(1-\ell) \varpi}{\max \{\lambda(\Lambda)\}},
\end{gathered}
$$

for $t \in\left[t_{i}, t_{i+1}\right), \ell \in(0,1)$, if matrices $\mathrm{H}, \Lambda$ and $\Gamma$ satisfy (13)-(16), and $\xi_{1}, \xi_{2}$, $\mu_{1}, \mu_{2}$ and $\varpi$ are the same as Theorem 1.

Proof. From (28)-(31), we get

$$
\dot{\sigma}_{5}(t) \geq-(1+\max \{\lambda(\mathrm{H})\}) \sigma_{5}(t)
$$

and

$$
\dot{\sigma}_{6}(t) \geq-(1+\max \{\lambda(\Lambda)\}) \sigma_{6}(t) .
$$

Then, we can obtain that $\sigma_{5}(t) \geq 0$ and $\sigma_{6}(t) \geq 0$.

We define the following function

$$
V_{2}(t)=V(t)+\sigma_{5}(t)+\sigma_{6}(t)
$$


where $V(t)$ is the same as (17).

For $t \in\left[t_{i}, t_{i+1}\right)$, we can get the upper right Dini-derivative of $V_{2}(t)$ as

$$
\begin{aligned}
& \dot{V}_{2}(t)=\dot{V}(t)+\dot{\sigma}_{5}(t)+\dot{\sigma}_{6}(t) \\
& \leq\left[-\min \{\lambda(W)\}+\max \{|\lambda(\tilde{C})|\}+\frac{M_{\max }}{1-\theta}\|\hat{\Omega}\|_{1}\right. \\
& \left.+M_{\max }\|\hat{\Delta}\|_{1}-\min \{\lambda(\mathrm{H})\}\right]\|e(t)\|_{1} \\
& +\max \{\lambda(\mathrm{H})\}\|\operatorname{me}(t)\|_{1}+[1-\min \{\lambda(\tilde{B})\}-\min \{\lambda(\Lambda)\}] \\
& \times\|r(t)\|_{1}+\max \{\lambda(\Lambda)\}\|m r(t)\|_{1}-\varpi \\
& -\sigma_{5}(t)+\xi_{1} \mu_{1}\|e(t)\|_{1}+\ell \varpi-\max \{\lambda(\mathrm{H})\}\|m e(t)\|_{1} \\
& -\sigma_{6}(t)+\xi_{2} \mu_{2}\|r(t)\|_{1}+(1-\ell) \varpi-\max \{\lambda(\Lambda)\}\|m r(t)\|_{1} \\
& =-\mu_{1}\|e(t)\|_{1}-\sigma_{5}(t)+\xi_{1} \mu_{1}\|e(t)\|_{1}-\mu_{2}\|r(t)\|_{1} \\
& -\sigma_{6}(t)+\xi_{2} \mu_{2}\|r(t)\|_{1} \\
& \leq\left(\xi_{1}-1\right) \mu_{1}\|e(t)\|_{1}+\left(\xi_{2}-1\right) \mu_{2}\|r(t)\|_{1}-\sigma_{5}(t)-\sigma_{6}(t) \\
& \leq 0,
\end{aligned}
$$

Therefore, IMNNs systems (2) and (3) can be synchronized with the state feedback controller (10) and the DETC conditions (30) and (31). The proof is finished.

Corollary 6. IMNNs systems (2) and (3) can be synchronized under assumptions 1 and 2 with the first type of state feedback controller (10) and the following DETC conditions

$$
\begin{gathered}
\|m e(t)\|_{1} \leq \frac{\max \{\lambda(\mathrm{H})\}}{\max \{\lambda(\mathrm{H})\}+\xi_{1} \mu_{1}} \sigma_{5}(t) \\
+\frac{\xi_{1} \mu_{1}\left\|e\left(t_{i}\right)\right\|_{1}+\ell \varpi}{\max \{\lambda(\mathrm{H})\}+\xi_{1} \mu_{1}},
\end{gathered}
$$

and

$$
\begin{gathered}
\|m r(t)\|_{1} \leq \frac{\max \{\lambda(\Lambda)\}}{\max \{\lambda(\Lambda)\}+\xi_{2} \mu_{2}} \sigma_{6}(t) \\
+\frac{\xi_{2} \mu_{2}\left\|r\left(t_{i}\right)\right\|_{1}+(1-\ell) \varpi}{\max \{\lambda(\Lambda)\}+\xi_{2} \mu_{2}},
\end{gathered}
$$

for $t \in\left[t_{i}, t_{i+1}\right), \ell \in(0,1)$, if matrices $\mathrm{H}, \Lambda$ and $\Gamma$ satisfy (13)-(16), and $\xi_{1}, \xi_{2}$, $\mu_{1}, \mu_{2}$ and $\varpi$ are the same as Theorem 1.

Proof. From (32) and (33), we can get

$$
\begin{aligned}
& \max \{\lambda(\mathrm{H})\}\|m e(t)\|_{1} \leq \max \{\lambda(\mathrm{H})\} \sigma_{5}(t) \\
& +\xi_{1} \mu_{1}\left(\left\|e\left(t_{i}\right)\right\|_{1}-\|m e(t)\|_{1}\right)+\ell \varpi \\
& \leq \max \{\lambda(\mathrm{H})\} \sigma_{5}(t)+\xi_{1} \mu_{1}\|e(t)\|_{1}+\ell \varpi,
\end{aligned}
$$


and

$$
\begin{aligned}
& \max \{\lambda(\Lambda)\}\|m r(t)\|_{1} \leq \max \{\lambda(\Lambda)\} \sigma_{6}(t) \\
& +\xi_{2} \mu_{2}\left(\left\|r\left(t_{i}\right)\right\|_{1}-\|m r(t)\|_{1}\right)+(1-\ell) \varpi \\
& \leq \max \{\lambda(\Lambda)\} \sigma_{6}(t)+\xi_{2} \mu_{2}\|r(t)\|_{1}+(1-\ell) \varpi,
\end{aligned}
$$

for $t \in\left[t_{i}, t_{i+1}\right)$, in other words, the inequalities (30) and (31) in Corollary 5 hold. Thus, all the conditions of Corollary 5 are satisfied.

Remark 6. By introducing two dynamic variables, DETC scheme can be obtained. It is very convenient to set DETC conditions due to the first type of state feedback controller (10). By analyzing SETC and DETC conditions 255 introduced in theorems 1-2 and corollaries 1-6, the construct of SETC and DETC is different. In SETC conditions, the thresholds of measured errors $m e(t)$ and $m r(t)$ are decided by $e(t)$ and $r(t)$ (or $e\left(t_{i}\right)$ and $r\left(t_{i}\right)$ ), respectively. While in DETC conditions, the thresholds of measured errors $m e(t)$ and $m r(t)$ are decided by $e(t), r(t)$ (or $e\left(t_{i}\right), r\left(t_{i}\right)$ ) and two dynamic variables. Compared with DETC scheme, SETC scheme is simpler. But DETC scheme is more flexible than SETC scheme. Moreover, the update times of controller under DETC scheme are less than that under SETC scheme, which will be verified in Section 4 .

\subsection{The Second Type of Controller}

Now, we discuss the second type of controller which is added to the original second-order system (4) or (5).

We consider the second type of state feedback controller in the Form (B) as follows

$$
v(t)=-\Lambda r\left(t_{i}\right)-\Gamma \operatorname{sgn}\left(r\left(t_{i}\right)\right), t \in\left[t_{i}, t_{i+1}\right)
$$

where $\Lambda=\operatorname{diag}\left(\Lambda_{1}, \Lambda_{2}, \ldots, \Lambda_{l}\right)^{T}$ is positive definite matrix; $\Gamma=\operatorname{diag}\left(\Gamma_{1}, \Gamma_{2}, \ldots, \Gamma_{l}\right)^{T}$; $\operatorname{sgn}()$ represents sign function; and $t_{i}$ is a release instant.

(1) Static Event-Triggered Control

Theorem 3. IMNNs systems (2) and (5) can be synchronized under assumptions 1 and 2 with the second type of state feedback controller (34) and the 
following SETC condition

$$
\|m r(t)\|_{1} \leq \xi_{2} \frac{\left(\mu_{2}\|r(t)\|_{1}+\varpi\right)}{\max \{\lambda(\Lambda)\}},
$$

for $t \in\left[t_{i}, t_{i+1}\right)$, if

$$
\min \{\lambda(W)\}>\max \{|\lambda(\tilde{C})|\}+\frac{M_{\max }}{1-\theta}\|\hat{\Omega}\|_{1}+M_{\max }\|\hat{\Delta}\|_{1},
$$

and inequalities (14)-(16) hold, and $\xi_{2}, \mu_{2}$ and $\varpi$ are the same as Theorem 1.

Proof. Consider a Lyapunov function $V(t)$ which is described in (17). For $t \in\left[t_{i}, t_{i+1}\right)$, we can get the upper right Dini-derivative of $V(t)$ as

$$
\begin{aligned}
& \dot{V}(t) \leq \operatorname{sgn}^{T}(e(t)) \dot{e}(t)+\operatorname{sgn}^{T}(r(t)) \dot{r}(t) \\
& +\sum_{m=1}^{l} \sum_{z=1}^{l} \hat{\beta}_{m z}\left[\frac{1}{1-\theta}\left|g_{z}\left(e_{z}(t)\right)\right|-\left|g_{z}\left(e_{z}\left(t-\tau_{m z}(t)\right)\right)\right|\right] \\
& =\operatorname{sgn}^{T}(e(t))\{-W e(t)+r(t)\} \\
& +\operatorname{sgn}^{T}(r(t))\{-\tilde{B} r(t)-\tilde{C} e(t)+\Delta(\tilde{p}(t)) g(e(t)) \\
& +(\Delta(\tilde{p}(t))-\Delta(p(t))) f(p(t)) \\
& +\Omega(\tilde{p}(t)) g(e(t-\tau(t)))+(\Omega(\tilde{p}(t))-\Omega(p(t))) \\
& \left.\times f(p(t-\tau(t)))-\Lambda r\left(t_{i}\right)-\Gamma \operatorname{sgn}\left(r\left(t_{i}\right)\right)\right\} \\
& +\sum_{m=1}^{l} \sum_{z=1}^{l} \hat{\beta}_{m z}\left[\frac{1}{1-\theta}\left|g_{z}\left(e_{z}(t)\right)\right|-\left|g_{z}\left(e_{z}\left(t-\tau_{m z}(t)\right)\right)\right|\right] \\
& \leq-\min \{\lambda(W)\}\|e(t)\|_{1}+\|r(t)\|_{1} \\
& -\min \{\lambda(\tilde{B})\}\|r(t)\|_{1}+\max \{|\lambda(\tilde{C})|\}\|e(t)\|_{1} \\
& +M_{\max }\|\hat{\Delta}\|_{1}\|e(t)\|_{1}-\min \{\lambda(\Lambda)\}\|r(t)\|_{1} \\
& +\max \{\lambda(\Lambda)\}\|m r(t)\|_{1}+\frac{M_{\max }}{1-\theta}\|\hat{\Omega}\|_{1}\|e(t)\|_{1} \\
& -\sum_{m=1}^{l}\left\{\kappa_{m}-\sum_{z=1}^{l}\left[\left|\vec{\alpha}_{m z}-\overleftarrow{\alpha}_{m z}\right|+\left|\vec{\beta}_{m z}-\overleftarrow{\beta}_{m z}\right|\right] N_{z}\right\} \\
& =\left[-\min \{\lambda(W)\}+\max \{|\lambda(\tilde{C})|\}+M_{\max }\|\hat{\Delta}\|_{1}\right. \\
& \left.+\frac{M_{\max }}{1-\theta}\|\hat{\Omega}\|_{1}\right]\|e(t)\|_{1}+[1-\min \{\lambda(\tilde{B})\}-\min \{\lambda(\Lambda)\}] \\
& \times\|r(t)\|_{1}+\max \{\lambda(\Lambda)\}\|m r(t)\|_{1}-\varpi \\
& \leq\left(\xi_{2}-1\right)\left(\mu_{2}\|r(t)\|_{1}+\varpi\right) \leq 0
\end{aligned}
$$

Therefore, IMNNs systems (2) and (5) are synchronized. The proof is completed. 
To achieve synchronization of IMNNs, the derivative of Lyapunov function needs to be not more than 0 . Therefore, we can have $\xi_{1} \in[0,1]$ and $\xi_{2} \in[0,1]$, which are described in all theorems and corollaries. For example, in the proof of Theorem 3, some conditions are provided to make the following inequality

$$
\dot{V}(t) \leq\left(\xi_{2}-1\right)\left(\mu_{2}\|r(t)\|_{1}+\varpi\right)
$$

hold. Therefore, $\xi_{2} \leq 1$ such that $\dot{V}(t) \leq 0$. In addition, the SETC condition in Theorem 3 is given by

$$
\|m r(t)\|_{1} \leq \xi_{2} \frac{\left(\mu_{2}\|r(t)\|_{1}+\varpi\right)}{\max \{\lambda(\Lambda)\}} .
$$

This means that $\xi_{2} \geq 0$. Thus, we can get $\xi_{2} \in[0,1]$. The similar results for $\xi_{1} \in[0,1]$ and $\xi_{2} \in[0,1]$ in other theorems and corollaries can be obtained. Therefore, Table 1 and Table 2 in the Section 4 show the data transmission rate with $\xi_{2} \in[0,1]$.

Corollary \%. IMNNs systems (2) and (5) can be synchronized under assumptions 1 and 2 with the second type of state feedback controller (34) and the following SETC condition

$$
\|m r(t)\|_{1} \leq \frac{\xi_{2}\left(\mu_{2}\left\|r\left(t_{i}\right)\right\|_{1}+\varpi\right)}{\max \{\lambda(\Lambda)\}+\xi_{2} \mu_{2}}
$$

for $t \in\left[t_{i}, t_{i+1}\right)$, if inequalities (14)-(16) and (36) hold, and $\xi_{2}, \mu_{2}$ and $\varpi$ are the same as Theorem 1 .

\section{(2) Dynamic Event-Triggered Control}

Theorem 4. IMNNs systems (2) and (5) can be synchronized under assumptions 1 and 2 with the second type of state feedback controller (34) and the following DETC condition

$$
\|m r(t)\|_{1} \leq \sigma_{2}(t)+\xi_{2} \frac{\left(\mu_{2}\|r(t)\|_{1}+\varpi\right)}{\max \{\lambda(\Lambda)\}}
$$

for $t \in\left[t_{i}, t_{i+1}\right)$, if inequalities (14)-(16) and (36) hold, $\xi_{2}, \mu_{2}$ and $\varpi$ are the same as Theorem 1, dynamic variable $\sigma_{2}(t)$ satisfies the condition (25).

Corollary 8. IMNNs systems (2) and (5) can be synchronized under assumptions 1 and 2 with the second type of state feedback controller (34) and 
the following DETC condition

$$
\begin{gathered}
\|m r(t)\|_{1} \leq \frac{\max \{\lambda(\Lambda)\}}{\max \{\lambda(\Lambda)\}+\xi_{2} \mu_{2}} \sigma_{2}(t) \\
+\xi_{2} \frac{\left(\mu_{2}\left\|r\left(t_{i}\right)\right\|_{1}+\varpi\right)}{\max \{\lambda(\Lambda)\}+\xi_{2} \mu_{2}},
\end{gathered}
$$

for $t \in\left[t_{i}, t_{i+1}\right)$, if inequalities (14)-(16) and (36) hold, $\xi_{2}, \mu_{2}$ and $\varpi$ are the same as Theorem 1, dynamic variable $\sigma_{2}(t)$ satisfies the condition (25).

In this paper, four theorems and eight corollaries are proposed, and the event-triggered control condition has many forms. The basis for these forms of event-triggered control conditions is to make that Lyapunov function will be not more than 0 . We take Theorem 1 and Corollary 1 as examples to explain the basis of these forms.

The purpose of Theorem 1 and Corollary 1 is to let $\dot{V}(t) \leq 0$. Thus, we can set different ETC schemes to make $\dot{V}(t) \leq 0$.

Then the SETC conditions (11) and (12) in Theorem 1 are built, such that

$$
\dot{V}(t) \leq\left(\xi_{1}-1\right) \mu_{1}\|e(t)\|_{1}+\left(\xi_{2}-1\right)\left(\mu_{2}\|r(t)\|_{1}+\varpi\right) .
$$

Similarly, the SETC conditions (18) and (19) in Corollary 1 are structured, such that

$$
\dot{V}(t) \leq\left(\xi_{1}-1\right)\left(\mu_{1}\|e(t)\|_{1}+\varpi\right)+\left(\xi_{2}-1\right) \mu_{2}\|r(t)\|_{1} .
$$

Finally, by using $\xi_{1}, \xi_{2} \in[0,1]$, we can obtain $\dot{V}(t) \leq 0$.

Remark \%. Different from the first type of state feedback controller (10), the second type of controller (34) is added to the original second-order system. The two types of controllers have their respective advantages. On one hand, it is more convenient to set SETC and DETC conditions under the first type of controller than that under the second type of controller. On the other hand, the second type of controller is simpler than the first type of controller. According to practical needs, we can adopt appropriate controller.

Remark 8. ETC schemes have been widely used in the first-order systems [42-50]. Compared with the existing ETC methods [42-50], the proposed ETC schemes have two significant advantages: 1) Wider application - The proposed ETC schemes can be used in the second-order systems besides the first-order 
systems; 2) More flexible - Due to more chances and choices to set ETC conditions, our ETC schemes are more convenient and flexible.

Remark 9. If there exists a finite constant $Q$ such that

$$
\lim _{i \rightarrow \infty} t_{i}=\sum_{i=0}^{\infty}\left(t_{i+1}-t_{i}\right)=Q,
$$

then system exhibits Zeno behavior [51]. Nevertheless, Zeno behavior is not desired in system. When event-triggered release times in finite time is finite, namely, execution time $\bar{t}_{i}=t_{i+1}-t_{i}$ is bigger than a positive constant, then Zeno behavior will not occur in the system. Actually, all SETC and DETC conditions provided in these theorems and corollaries of this paper will not make IMNNs exhibit Zeno behavior. We take Theorem 3 as an example. In Theorem 3, when event is triggered for $t \in\left[t_{i}, t_{i+1}\right)$, we can have

$$
\left\|m r\left(t_{i+1}\right)\right\|_{1}>\xi_{2} \frac{\left(\mu_{2}\|r(t)\|_{1}+\varpi\right)}{\max \{\lambda(\Lambda)\}},
$$

In addition,

$$
\begin{aligned}
& \frac{\mathrm{d}}{\mathrm{d} t}\|m r(t)\|_{1} \leq\left\|\frac{\mathrm{d}}{\mathrm{d} t} m r(t)\right\|_{1}=\|\dot{r}(t)\|_{1} \\
& =\|-\tilde{B} r(t)-\tilde{C} e(t)+\Delta(\tilde{p}(t)) f(\tilde{p}(t)) \\
& -\Delta(p(t)) f(p(t))+\Omega(\tilde{p}(t)) f(\tilde{p}(t-\tau(t))) \\
& -\Omega(p(t)) f(p(t-\tau(t)))-\Lambda r\left(t_{i}\right)-\Gamma \operatorname{sgn}\left(r\left(t_{i}\right)\right) \|_{1} \\
& \leq\|\tilde{B}\|_{1}\|r(t)\|_{1}+\|\tilde{C}\|_{1}\|e(t)\|_{1}+2\left(\|\hat{\Delta}\|_{1}+\|\hat{\Omega}\|_{1}\right) M_{\max } \\
& +\|\Lambda\|_{1}\left\|r\left(t_{i}\right)\right\|_{1}+\|\Gamma\|_{1} \\
& \leq\|\tilde{B}\|_{1}\|m r(t)\|_{1}+\left(\|\tilde{B}\|_{1}+\|\Lambda\|_{1}\right)\left\|r\left(t_{i}\right)\right\|_{1}+\|\Gamma\|_{1} \\
& +\|\tilde{C}\|_{1}\|e(t)\|_{1}+2\left(\|\hat{\Delta}\|_{1}+\|\hat{\Omega}\|_{1}\right) M_{\max }
\end{aligned}
$$

Combining with $\dot{V}(t) \leq 0$ and the expression of $V(t)$, we can get

$$
\|e(t)\|_{1} \leq V(0)
$$

and

$$
\|r(t)\|_{1} \leq V(0) .
$$

Let

$$
\begin{aligned}
& J=\left(\|\tilde{B}\|_{1}+\|\Lambda\|_{1}+\|\tilde{C}\|_{1}\right) V(0)+\|\Gamma\|_{1} \\
& +2\left(\|\hat{\Delta}\|_{1}+\|\hat{\Omega}\|_{1}\right) M_{\max } .
\end{aligned}
$$


Then,

$$
\frac{\mathrm{d}}{\mathrm{d} t}\|m r(t)\|_{1} \leq\|\tilde{B}\|_{1}\|m r(t)\|_{1}+J .
$$

Because $m r\left(t_{i}\right)=0$, we can have

$$
\|m r(t)\|_{1} \leq \frac{J}{\|\tilde{B}\|_{1}}\left[e^{\|\tilde{B}\|_{1}\left(t-t_{i}\right)}-1\right]
$$

for $t \in\left[t_{i}, t_{i+1}\right)$. So,

$$
\xi_{2} \frac{\left(\mu_{2}\|r(t)\|_{1}+\varpi\right)}{\max \{\lambda(\Lambda)\}}<\left\|m r\left(t_{i+1}\right)\right\|_{1} \leq \frac{J}{\|\tilde{B}\|_{1}}\left[e^{\|\tilde{B}\|_{1}\left(t_{i+1}-t_{i}\right)}-1\right]
$$

and

$$
\begin{aligned}
& \bar{t}_{i}=t_{i+1}-t_{i}>\frac{1}{\|\tilde{B}\|_{1}} \ln \left[\frac{\xi_{2}\|\tilde{B}\|_{1}\left(\mu_{2}\|r(t)\|_{1}+\varpi\right)}{\max \{\lambda(\Lambda)\} J}+1\right] \\
& \geq \frac{1}{\|\tilde{B}\|_{1}} \ln \left[\frac{\xi_{2}\|\tilde{B}\|_{1} \varpi}{\max \{\lambda(\Lambda)\} J}+1\right] .
\end{aligned}
$$

Therefore, under the conditions of Theorem 3, error system (8) (or (9)) will not exhibit Zeno behavior.

\section{Numerical Simulation and Data Analyses}

In this section, we provide an example and some data analyses to verify the validity of the obtained results.

Example. Consider a drive IMNN as

$$
\begin{aligned}
& \frac{d^{2} p_{m}(t)}{d t^{2}}=-b_{m} \frac{d p_{m}(t)}{d t}-c_{m} p_{m}(t)+\sum_{z=1}^{2} \alpha_{m z}\left(p_{m}(t)\right) \\
& \times f_{z}\left(p_{z}(t)\right)+\sum_{z=1}^{2} \beta_{m z}\left(p_{m}(t)\right) f_{z}\left(p_{z}\left(t-\tau_{m z}(t)\right)\right) \\
& +I_{m}(t), m=1,2
\end{aligned}
$$

where $b_{1}=b_{2}=4.5 ; c_{1}=c_{2}=1.8 ; \tau_{m z}(t)=0.1 \sin (t), m, z=1,2$; external input $I_{1}(t)=I_{2}(t)=0$; memristive connection weights:

$$
\begin{gathered}
\alpha_{11}\left(p_{1}(t)\right)= \begin{cases}1.2, & \left|p_{1}(t)\right| \leq 1, \\
0.9, & \left|p_{1}(t)\right|>1,\end{cases} \\
\alpha_{12}\left(p_{1}(t)\right)= \begin{cases}-0.3, & \left|p_{1}(t)\right| \leq 1, \\
-0.16, & \left|p_{1}(t)\right|>1,\end{cases}
\end{gathered}
$$




$$
\begin{aligned}
& \alpha_{21}\left(p_{2}(t)\right)= \begin{cases}-0.5, & \left|p_{2}(t)\right| \leq 1, \\
-0.8, & \left|p_{2}(t)\right|>1,\end{cases} \\
& \alpha_{22}\left(p_{2}(t)\right)= \begin{cases}0.09, & \left|p_{2}(t)\right| \leq 1, \\
0.25, & \left|p_{2}(t)\right|>1,\end{cases} \\
& \beta_{11}\left(p_{1}(t)\right)= \begin{cases}-0.48, & \left|p_{1}(t)\right| \leq 1, \\
-0.34, & \left|p_{1}(t)\right|>1,\end{cases} \\
& \beta_{12}\left(p_{1}(t)\right)= \begin{cases}0.36, & \left|p_{1}(t)\right| \leq 1, \\
0.56, & \left|p_{1}(t)\right|>1,\end{cases} \\
& \beta_{21}\left(p_{2}(t)\right)= \begin{cases}0.65, & \left|p_{2}(t)\right| \leq 1, \\
0.8, & \left|p_{2}(t)\right|>1,\end{cases} \\
& \beta_{22}\left(p_{2}(t)\right)= \begin{cases}0.6, & \left|p_{2}(t)\right| \leq 1, \\
0.4, & \left|p_{2}(t)\right|>1,\end{cases}
\end{aligned}
$$

Then, we can get that

$$
\begin{aligned}
& \hat{\Delta}=\left[\begin{array}{cc}
1.2 & 0.3 \\
0.8 & 0.25
\end{array}\right], \\
& \hat{\Omega}=\left[\begin{array}{cc}
0.48 & 0.56 \\
0.8 & 0.6
\end{array}\right],
\end{aligned}
$$

and $\|\hat{\Delta}\|=2,\|\hat{\Omega}\|=1.28$.

Set $\omega_{1}=\omega_{2}=4$ and $q_{m}(t)=\frac{d p_{m}(t)}{d t}+4 p_{m}(t), m=1,2$. Then system (39) can be rewritten as

$$
\left\{\begin{array}{l}
\frac{d p_{1}(t)}{d t}=-4 p_{1}(t)+q_{1}(t) \\
\frac{d p_{2}(t)}{d t}=-4 p_{2}(t)+q_{2}(t) \\
\frac{d q_{1}(t)}{d t}=-0.5 q_{1}(t)+0.2 p_{1}(t)+\sum_{z=1}^{2} \alpha_{1 z}\left(p_{1}(t)\right) \\
\times f_{z}\left(p_{z}(t)\right)+\sum_{z=1}^{2} \beta_{1 z}\left(p_{1}(t)\right) f_{z}\left(p_{z}(t-1)\right), \\
\frac{d q_{2}(t)}{d t}=-0.5 q_{2}(t)+0.2 p_{2}(t)+\sum_{z=1}^{2} \alpha_{2 z}\left(p_{2}(t)\right) \\
\times f_{z}\left(p_{z}(t)\right)+\sum_{z=1}^{2} \beta_{2 z}\left(p_{2}(t)\right) f_{z}\left(p_{z}(t-1)\right),
\end{array}\right.
$$


We consider the second type of controller, therefore, the corresponding response system (Form (B)) can be written as

$$
\left\{\begin{array}{l}
\frac{d \tilde{p}_{1}(t)}{d t}=-4 \tilde{p}_{1}(t)+\tilde{q}_{1}(t), \\
\frac{d \tilde{p}_{2}(t)}{d t}=-4 \tilde{p}_{2}(t)+\tilde{q}_{2}(t), \\
\frac{d \tilde{q}_{1}(t)}{d t}=-0.5 \tilde{q}_{1}(t)+0.2 \tilde{p}_{1}(t)+\sum_{z=1}^{2} \alpha_{1 z}\left(\tilde{p}_{1}(t)\right) \\
\times f_{z}\left(\tilde{p}_{z}(t)\right)+\sum_{z=1}^{2} \beta_{1 z}\left(\tilde{p}_{1}(t)\right) f_{z}\left(\tilde{p}_{z}(t-1)\right)+v_{1}(t), \\
\frac{d \tilde{q}_{2}(t)}{d t}=-0.5 \tilde{q}_{2}(t)+0.2 \tilde{p}_{2}(t)+\sum_{z=1}^{2} \alpha_{2 z}\left(\tilde{p}_{2}(t)\right) \\
\times f_{z}\left(\tilde{p}_{z}(t)\right)+\sum_{z=1}^{2} \beta_{2 z}\left(\tilde{p}_{2}(t)\right) f_{z}\left(\tilde{p}_{z}(t-1)\right)+v_{2}(t),
\end{array}\right.
$$

Considering activation function $f_{z}(x)=\frac{|x+1|-|x-1|}{2}$, we can obtain that $M_{z}=1, N_{z}=1, z=1,2$. Combining with

$$
\sum_{z=1}^{2}\left[\left|\vec{\alpha}_{1 z}-\overleftarrow{\alpha}_{1 z}\right|+\left|\vec{\beta}_{1 z}-\overleftarrow{\beta}_{1 z}\right|\right] N_{z}=0.78
$$

and

$$
\sum_{z=1}^{2}\left[\left|\vec{\alpha}_{2 z}-\overleftarrow{\alpha}_{2 z}\right|+\left|\vec{\beta}_{2 z}-\overleftarrow{\beta}_{2 z}\right|\right] N_{z}=0.81
$$

we can set $\kappa_{1}=0.8, \kappa_{2}=0.83, \varpi=0.04$. Then, we set $\Gamma$ as follows

$$
\left\{\begin{array}{l}
\Gamma_{1}=0.82, \quad \text { if } \operatorname{sgn}\left(r_{1}(t)\right) \operatorname{sgn}\left(r_{1}\left(t_{i}\right)\right)>0 \\
\Gamma_{1}=-0.82, \quad \text { otherwise }
\end{array}\right.
$$

and

$$
\left\{\begin{array}{l}
\Gamma_{2}=0.85, \quad \text { if } \operatorname{sgn}\left(r_{2}(t)\right) \operatorname{sgn}\left(r_{2}\left(t_{i}\right)\right)>0, \\
\Gamma_{2}=-0.85, \quad \text { otherwise }
\end{array}\right.
$$

Choosing $\Lambda=\operatorname{diag}\{0.75,2\}$, we can get that $\mu_{2}=0.25$.

Therefore, we can obtain the following SETC and DETC conditions.

1) SETC condition (Theorem 3):

$$
\|m r(t)\|_{1} \leq \xi_{2}\left(0.125\|r(t)\|_{1}+0.02\right)
$$

2) DETC condition (Theorem 4):

$$
\|m r(t)\|_{1} \leq \sigma_{2}(t)+\xi_{2}\left(0.125\|r(t)\|_{1}+0.02\right),
$$




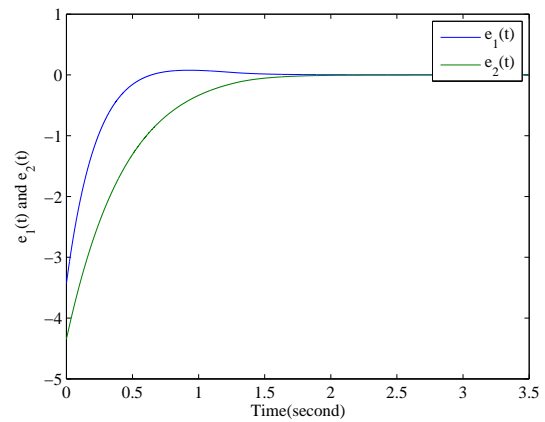

Fig. 1. Synchronization errors $e_{1}(t)$ and $e_{2}(t)$ of systems under SETC condition with the second type of controller and $\xi_{2}=0.5$.

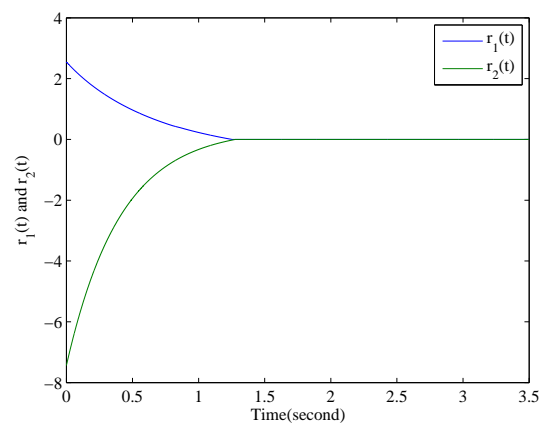

Fig. 2. Synchronization errors $r_{1}(t)$ and $r_{2}(t)$ of systems under SETC condition with the second type of controller and $\xi_{2}=0.5$.

for $t \in\left[t_{i}, t_{i+1}\right), \xi_{2} \in[0,1]$, where $\dot{\sigma}_{2}(t)=-\sigma_{2}(t)+\xi_{2}\left(0.25\|r(t)\|_{1}+0.04\right)-$ $3152\|m r(t)\|_{1}$, the initial value satisfies $\sigma_{2}(0) \geq 0$.

From the conditions of Theorems 3 and 4, we can get that the IMNN systems (40) and (41) can be synchronized with the second type of controller under the SETC condition (42) and DETC condition (43).

As shown in Figs. 1, 7 and 2, 8, errors $e_{m}(t)$ and $r_{m}(t), m=1,2$ converge to zero asymptotically under SETC and DETC condition. Sample error $r_{m}\left(t_{i}\right)$ and measured error $m r_{m}(t)$ are shown in Figs. 3, 9 and 4, 10, respectively. Before measured error $m r_{m}(t)$ breaches the SETC or DETC condition, sample error $r_{m}\left(t_{i}\right)$ remains unchanged. When measured error $m r_{m}(t)$ breaches the SETC 


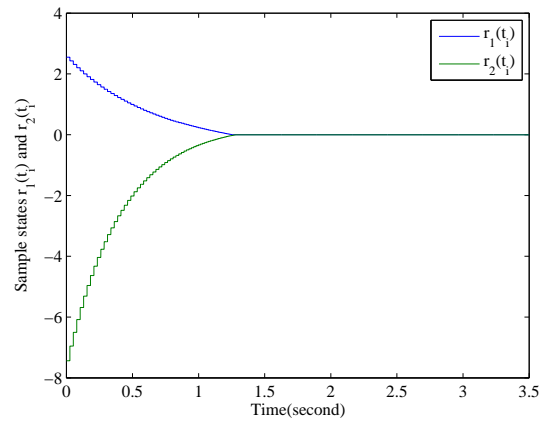

Fig. 3. Sample errors $r_{1}\left(t_{i}\right)$ and $r_{2}\left(t_{i}\right)$ of systems under SETC condition with the second type of controller and $\xi_{2}=0.5$.

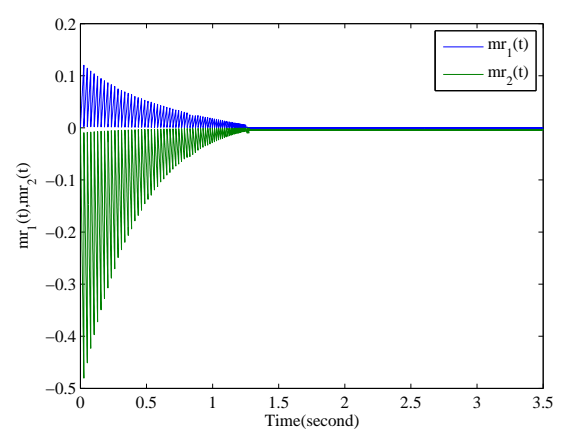

Fig. 4. Measured errors $m r_{1}(1)$ and $m r_{2}(t)$ of systems under SETC condition with the second type of controller and $\xi_{2}=0.5$.

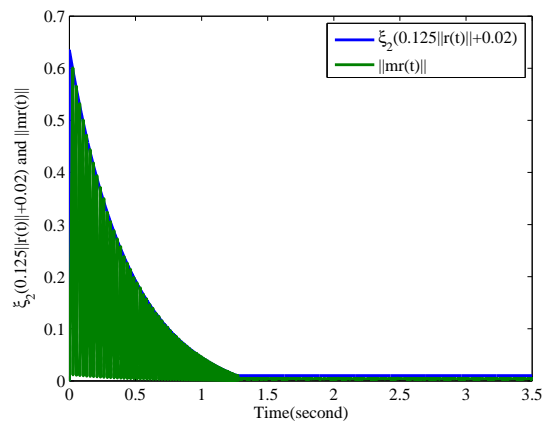

Fig. 5. 1-norm $\|m r(t)\|_{1}$ and the threshold $\xi_{2}\left(0.125\|r(t)\|_{1}+0.02\right)$ under SETC condition with the second type of controller and $\xi_{2}=0.5$. 


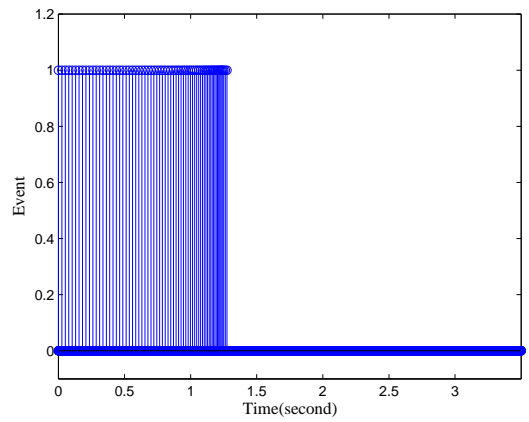

Fig. 6. Event-triggered instants under SETC condition with the second type of controller and $\xi_{2}=0.5$.

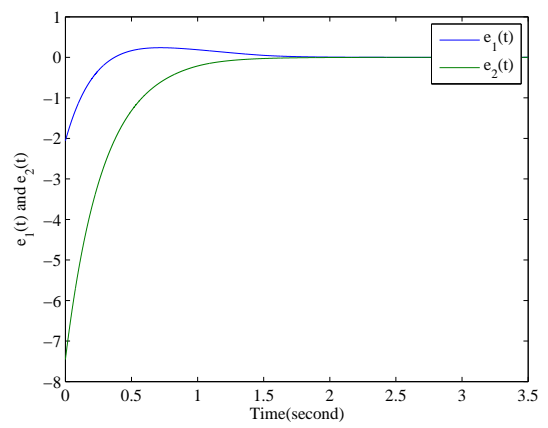

Fig. 7. Synchronization errors $e_{1}(t)$ and $e_{2}(t)$ of systems under DETC condition with the second type of controller and $\xi_{2}=0.5$.

or DETC condition, in other words, 1-norm $\|m r(t)\|_{1}$ exceeds the threshold $\xi_{2}\left(0.125\|r(t)\|_{1}+0.02\right)$ for SETC condition and $\sigma_{2}(t)+\xi_{2}\left(0.125\|r(t)\|_{1}+0.02\right)$ for DETC condition, the event is triggered, as shown in Figs. 5, 6, 11 and 12. In this paper, IMNN systems (41) and (40) can be synchronized and effectively reduce the update times of controller via SETC or DETC scheme.

Now, we discuss the relationship between parameter $\xi_{2}$ and trigger frequency. 330 There are three performance parameters (trigger times $T_{t}$, data transmission rate $T_{r}$ and average release period $R_{p}$ ) which will be used. Table 1 shows that there are just 154 trigger times and $7.70 \%$ data transmission rate when we set $\xi_{2}=0.2$ under SETC strategy. In other words, the controller just updates 154 


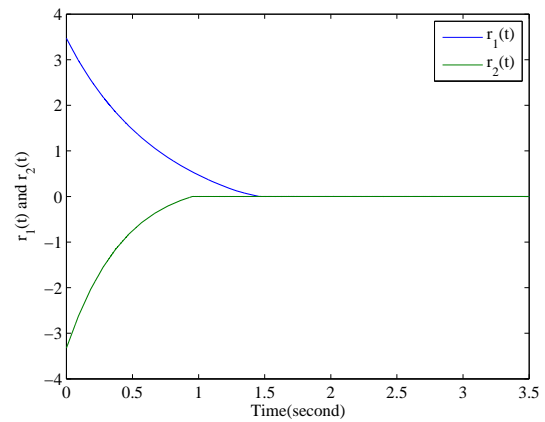

Fig. 8. Synchronization errors $r_{1}(t)$ and $r_{2}(t)$ of systems under DETC condition with the second type of controller and $\xi_{2}=0.5$.

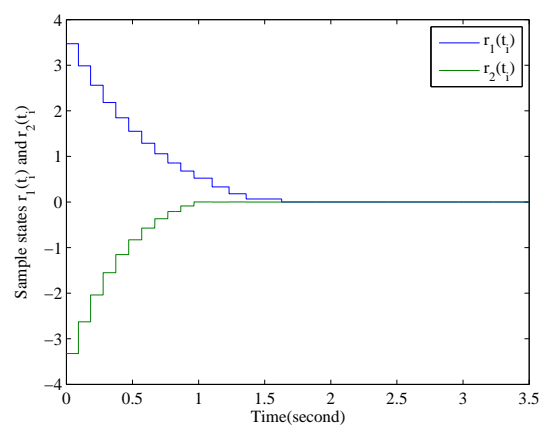

Fig. 9. Sample errors $r_{1}\left(t_{i}\right)$ and $r_{2}\left(t_{i}\right)$ of systems under DETC condition with the second type of controller and $\xi_{2}=0.5$.

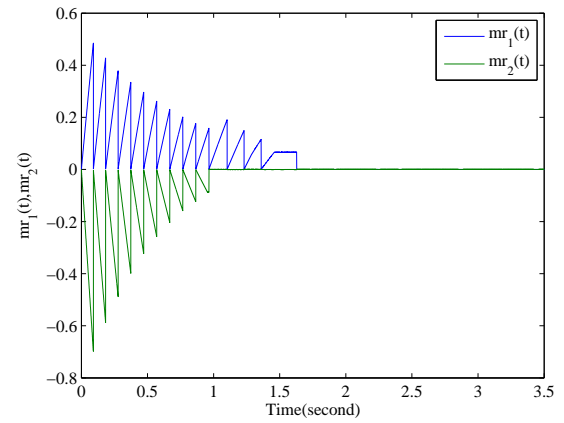

Fig. 10. Measured errors $m r_{1}(1)$ and $m r_{2}(t)$ of systems under DETC condition with the second type of controller and $\xi_{2}=0.5$. 


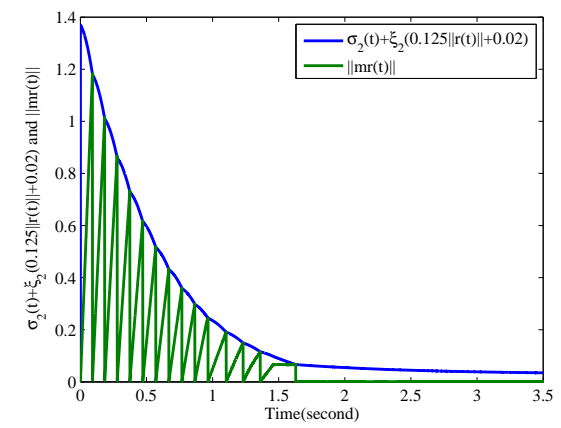

Fig. 11. 1-norm $\|m r(t)\|_{1}$ and the threshold $\sigma_{2}(t)+\xi_{2}\left(0.125\|r(t)\|_{1}+0.02\right)$ under DETC condition with the second type of controller and $\xi_{2}=0.5$.

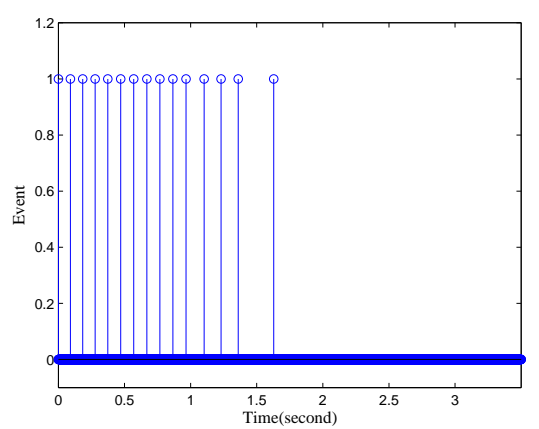

Fig. 12. Event-triggered instants under DETC condition with the second type of controller and $\xi_{2}=0.5$.

times, and saves $92.30 \%$ computational resource. As parameter $\xi_{2}$ is increased to 1 , the trigger times and data transmission rate are decreased to 31 and $1.55 \%$, respectively. Moreover, the increasing average release period means that trigger times will decrease when parameter $\xi_{2}$ is increased from 0 to 1 . Therefore, we can obtain that a large parameter $\xi_{2}$ under SETC strategy (42) can reduce computational burden and trigger frequency.

340 From Table 2, the controller updates 150 and 6 times, and saves $92.50 \%$ and $99.70 \%$ computational resource when $\xi_{2}$ is 0.2 and 1 , respectively. As parameter $\xi_{2}$ is increased, the trigger times and data transmission rate are decreased, and average release period is increased. Similarly, we can obtain 
Table 1. FOR FIXED TIME INTERVAL $h=0.0025, t=5$, PERFORMANCE PARAMETERS: $T_{t}, R_{p}$ AND $T_{r}$ UNDER THE SECOND TYPE OF CONTROLLER AND SETC CONDITION (42) WITH DIFFERENT $\xi_{2}$.

\begin{tabular}{c|c|c|c|c|c|c}
\hline$\xi_{2}$ & 0 & 0.2 & 0.4 & 0.6 & 0.8 & 1 \\
\hline$T_{t}$ & 2000 & 154 & 91 & 59 & 48 & 31 \\
\hline$R_{p}$ & 0.0025 & 0.0095 & 0.0202 & 0.0257 & 0.0491 & 0.0534 \\
\hline$T_{r}$ & $100 \%$ & $7.70 \%$ & $4.55 \%$ & $2.95 \%$ & $2.40 \%$ & $1.55 \%$ \\
\hline
\end{tabular}

Table 2. FOR FIXED TIME INTERVAL $h=0.0025, t=5$, PERFORMANCE PARAMETERS: $T_{t}, R_{p}$ AND $T_{r}$ UNDER THE SECOND TYPE OF CONTROLLER AND DETC CONDITION (43) WITH DIFFERENT $\xi_{2}$.

\begin{tabular}{c|c|c|c|c|c|c}
\hline$\xi_{2}$ & 0 & 0.2 & 0.4 & 0.6 & 0.8 & 1 \\
\hline$T_{t}$ & 878 & 150 & 63 & 12 & 9 & 6 \\
\hline$R_{p}$ & 0.0040 & 0.0119 & 0.0258 & 0.1204 & 0.1347 & 0.6698 \\
\hline$T_{r}$ & $43.90 \%$ & $7.50 \%$ & $3.15 \%$ & $0.60 \%$ & $0.45 \%$ & $0.30 \%$ \\
\hline
\end{tabular}

that a large parameter $\xi_{2}$ under DETC strategy (43) can reduce computational burden. For fixed parameter $\xi_{2}$, the trigger times and data transmission rate for DETC strategy are less than those for SETC strategy. Therefore, DETC strategy is a more appropriate way for achieving synchronization of IMNNs.

\section{Conclusion}

This paper discusses two types of state feedback controllers. The first type of controller is added to the transformational first-order system, the second type of controller is added to the original second-order system. Moreover, based on each controller, we study SETC and DETC strategies for synchronization of IMNNs with time-varying delays. Compared with traditional continuous-time control methods, ETC strategies can effectively reduce the update times of controller 

given to verify the validity of the obtained results.

In the future works, we will consider synchronization of various memristive neural networks via event-triggered control. Due to dependence on state for parameters of memristive neural networks and environment disturbances, there interesting to study synchronization of memristive neural networks under some parameter perturbations via event-triggered control.

\section{References}

[1] Y. Wang, T. Bian, J. Xiao, C. Wen, Global synchronization of complex dynamical networks through digital communication with limited data rate, IEEE Trans. Neural Netw. Learn. Syst. 26 (10) (2015) 2487-2499.

[2] W. Yang, Y. Wang, Y. Shen, L. Pan, Cluster synchronization of coupled delayed competitive neural networks with two time scales, Nonlinear Dyn. 90 (4) (2017) 2767-2782.

[3] J. Yi, Y. Wang, J. Xiao, Y. Huang, Synchronisation of complex dynamical networks with additive stochastic time-varying delays, Int. J. Syst. Sci. 47 (5) (2016) 1221-1229.

[4] L. Chen, Y. Wang, W. Yang, J. Xiao, Robust consensus of fractionalorder multi-agent systems with input saturation and external disturbances, Neurocomputing 303 (2018) 11-19.

[5] J. Yi, Y. Wang, J. Xiao, Y. Huang, Exponential synchronization of complex dynamical networks with markovian jump parameters and stochastic delays and its application to multi-agent systems, Commun. Nonlinear Sci. Numer. Simul. 18 (5) (2013) 1175-1192.

[6] M. Rubenstein, A. Cornejo, R. Nagpal, Programmable self-assembly in a thousand-robot swarm, Science 345 (2014) 795-799. 
[7] L. Shanmugam, P. Mani, R. Rajan, Y. Joo, Adaptive synchronization of reaction-diffusion neural networks and its application to secure communication, IEEE Trans. Cybern. doi: 10.1109/tcyb.2018.2877410.

[8] L. Zhou, C. Wang, S. Du, L. Zhou, Cluster synchronization on multiple nonlinearly coupled dynamical subnetworks of complex networks with nonidentical nodes, IEEE Trans. Neural Netw. Learn. Syst. 28 (3) (2017) 570583.

[9] J. Chen, K. Li, K. Bilal, X. Zhou, K. Li, P. Yu, A bi-layered parallel training architecture for large-scale convolutional neural networks, IEEE Trans. Parallel Distrib. Syst. 30 (5) (2019) 965-976.

[10] P. Melnyk, Z. You, K. Li, A high-performance CNN method for offline handwritten Chinese character recognition and visualization, Soft Comput. doi: 10.1007/s00500-019-04083-3.

[11] D. Strukov, G. Snider, D. Stewart, R. Williams, The missing memristor found, Nature 453 (2008) 80-83.

[12] L. Zhou, C. Wang, L. Zhou, A novel no-equilibrium hyperchaotic multiwing system via introducing memristor, Int. J. Circuit Theory Appl. 46 (1) (2018) 84-98.

[13] L. Zhou, C. Wang, L. Zhou, Generating hyperchaotic multi-wing attractor in a 4D memristive circuit, Nonlinear Dyn. 85 (4) (2016) 2653-2663.

[14] C. Wang, L. Xiong, J. Sun, W. Yao, Memristor-based neural networks with weight simultaneous perturbation training, Nonlinear Dyn. 95 (4) (2019) 2893-2906.

405

[15] L. Zhou, C. Wang, L. Zhou, Generating four-wing hyperchaotic attractor and two-wing, three-wing, and four-wing chaotic attractors in 4D memristive system, Int. J. Bifurc. Chaos 27 (2) (2017) 1750027-1-1750027-14. 
[16] C. Wang, X. Liu, H. Xia, Multi-piecewise quadratic nonlinearity memristor and its $2 \mathrm{~N}$-scroll and 2N+1-scroll chaotic attractors system, Chaos 27 (3) (2017) 033114-1-033114-12.

[17] M. Itoh, L. Chua, Memristor cellular automata and memristor discrete-time cellular neural networks, Int. J. Bifurc. Chaos 19 (11) (2009) 3605-3656.

[18] S. Duan, X. Hu, Z. Dong, L. Wang, P. Mazumder, Memristor-based cellular nonlinear/neural network: design, analysis, and applications, IEEE Trans. Neural Netw. Learn. Syst. 26 (6) (2015) 1202-1213.

[19] A. Wu, S. Wen, Z. Zeng, Synchronization control of a class of memristorbased recurrent neural networks, Inf. Sci. 183 (1) (2012) 106-116.

[20] S. Wen, G. Bao, Z. Zeng, et al. Global exponential synchronization of memristor-based recurrent neural networks with time-varying delays, Neural Netw. 48 (2013) 195-203.

[21] W. Zhang, C. Li, T. Huang, et al. Synchronization of memristor-based coupling recurrent neural networks with time-varying delays and impulses, IEEE Trans. Neural Netw. Learn. Syst. 26 (12) (2015) 3308-3313.

[22] N. Li, J. Cao, Lag synchronization of memristor-based coupled neural networks via $\omega$-measure, IEEE Trans. Neural Netw. Learn. Syst. 27 (3) (2016) 686-697.

[23] H. Bao, H. Ju, J. Cao, Adaptive synchronization of fractional-order memristor-based neural networks with time delay, Nonlinear Dyn. 82 (3) (2015) 1343-1354.

[24] L. Wang, Y. Shen, Q. Yin, et al. Adaptive synchronization of memristorbased neural networks with time-varying delays, IEEE Trans. Neural Netw. Learn. Syst. 26 (9) (2015) 2033-2042.

[25] Y. Wang, W. Yang, J. Xiao, Z. Zeng, Impulsive multisynchronization of coupled multistable neural networks with time-varying delay, IEEE Trans. Neural Netw. Learn. Syst. 28(7) (2017) 1560-1571. 
[26] X. Lv, X. Li, J. Cao, et al. Dynamical and static multisynchronization of coupled multistable neural networks via impulsive control, IEEE Trans. Neural Netw. Learn. Syst. 29 (12) (2018) 6062-6072.

[27] W. Yao, C. Wang, J. Cao, Y. Sun, C. Zhou, Hybrid multisynchronization of coupled multistable memristive neural networks with time delays, Neurocomputing 363 (21) (2019) 281-294.

[28] L. Wang, Z. Zeng, M. Ge, J. Hu, Global stabilization analysis of inertial memristive recurrent neural networks with discrete and distributed delays, Neural Netw. 105 (2018) 65-74.

[29] L. Wang, M. Ge, J. Hu, G. Zhang, Global stability and stabilization for inertial memristive neural networks with unbounded distributed delays, Nonlinear Dyn. 95 (2) (2019) 943-955.

[30] R. Rakkiyappan, S. Premalatha, A. Chandrasekar, J. Cao, Stability and synchronization analysis of inertial memristive neural networks with time delays, Cogn. Neurodyn. 10 (5) (2016) 437-451.

[31] R. Rakkiyappan, E. Udhaya Kumari, A. Chandrasekar, R. Krishnasamy, Synchronization and periodicity of coupled inertial memristive neural networks with supremums, Neurocomputing 214 (2016) 739-749.

[32] Z. Guo, S. Gong, T. Huang, Finite-time synchronization of inertial memristive neural networks with time delay via delay-dependent control, Neurocomputing 293 (2018) 100-107.

[33] A. Alimi, C. Aouiti, E. Assali, Finite-time and fixed-time synchronization of a class of inertial neural networks with multi-proportional delays and its application to secure communication, Neurocomputing doi: 10.1016/j.neucom.2018.11.020.

[34] R. Wei, J. Cao, A. Alsaedi, Finite-time and fixed-time synchronization analysis of inertial memristive neural networks with time-varying delays, Cogn. Neurodyn. 12 (1) (2017) 121-134. 
[35] D. Huang, M. Jiang, J. Jian, Finite-time synchronization of inertial memristive neural networks with time-varying delays via sampled-date control, Neurocomputing 266 (2017) 527-539.

[36] S. Gong, S. Yang, Z. Guo, T. Huang, Global exponential synchronization of inertial memristive neural networks with time-varying delay via nonlinear controller, Neural Netw. 102 (2018) 138-148.

[37] Z. Guo, S. Gong, S. Yang, T. Huang, Global exponential synchronization of multiple coupled inertial memristive neural networks with time-varying delay via nonlinear coupling, Neural Netw. doi: 10.1016/j.neunet.2018.08.020.

[38] P. Antsaklis, J. Baillieul, Special issue on technology of networked control systems, Proc. IEEE 95 (1) (2007) 5-8.

[39] T. Yang, Networked control system: a brief survey, IEE Proc. Contr. Theory Appl. 153 (4) (2006) 403-412.

[40] G. Leen, D. Heffernan, Time-triggeresd controller area network, Comput. Control Eng. J. 12 (6) (2001) 245-256.

[41] T. Nghiem, G. Pappas, R. Alur, A. Girard, Time-triggered implementations of dynamic controllers, ACM Trans. Embed. Comput. Syst. 11 (2012) 58.

[42] J. Zhou, H. Dong, J. Feng, Event-triggered communication for synchronization of Markovian jump delayed complex networks with partially unknown transition rates, Appl. Math. Comput. 293 (2017) 617-629.

[43] S. Wen, Z. Zeng, M. Chen, T. Huang, Synchronization of switched neural networks with communication delays via the event-triggered control, IEEE Trans. Neural Netw. Learn. Syst. 28 (10) (2017) 2334-2343.

[44] D. Yue, E. Tian, Q. Han, A delay system method for designing eventtriggered controllers of networked control systems, IEEE Trans. Automat. Contr. 58 (2) (2013) 475-481. 
[51] A. Ames, S. Sastry, Characterization of Zeno behavior in hybrid systems using homological methods, in Proc. Amer. Control Conf. 2 (2005) 11601165 . stability and stabilization of memristive neural networks with communication delays, Appl. Math. Comput. 310 (2017) 57-74.

[46] Y. Zhou, Z. Zeng, Event-triggered impulsive control on quasisynchronization of memristive neural networks with time-varying delays, Neural Netw. 110 (2019) 55-65.

[47] H. Liu, Z. Wang, B. Shen, X. Liu, Event-triggered $H_{\infty}$ state estimation for delayed stochastic memristive neural networks with missing measurements: the discrete time case, IEEE Trans. Neural Netw. Learn. Syst. 29 (8) (2018) 3726-3737.

[48] Z. Guo, S. Gong, S. Wen, T. Huang, Event-based synchronization control for memristive neural networks with time-varying delay, IEEE Trans. Cybern. 49 (9) (2019) 3268-3277.

[49] Y. Wang, Y. Lei, T. Bian, Z. Guan, Distributed control of nonlinear multi-agent systems with unknown and nonidentical control directions via event-triggered communication, IEEE Trans. Cybern. doi: 10.1109/TCYB.2019.2908874.

[50] X. Xie, Q. Zhou, D. Yue, H. Li, Relaxed control design of discrete-time Takagi-Sugeno fuzzy systems: an event-triggered real-time scheduling approach, IEEE Trans. Syst. Man Cybern. Syst. 48 (12) (2018) 2251-2262. 